\title{
High-Order Semi-Discrete Central-Upwind Schemes for Multi-Dimensional Hamilton-Jacobi Equations
}

\author{
Steve Bryson* Doron Levy ${ }^{\dagger}$
}

August 8, 2002

\begin{abstract}
We present the first fifth-order, semi-discrete central-upwind method for approximating solutions of multi-dimensional Hamilton-Jacobi equations. Unlike most of the commonly used high-order upwind schemes, our scheme is formulated as a Godunov-type scheme. The scheme is based on the fluxes of KurganovTadmor and Kurganov-Tadmor-Petrova, and is derived for an arbitrary number of space dimensions. A theorem establishing the monotonicity of these fluxes is provided. The spacial discretization is based on a weighted essentially nonoscillatory reconstruction of the derivative. The accuracy and stability properties of our scheme are demonstrated in a variety of examples. A comparison between our method and other fifth-order schemes for Hamilton-Jacobi equations shows that our method exhibits smaller errors without any increase in the complexity of the computations.
\end{abstract}

Key words. Hamilton-Jacobi equations, central schemes, semi-discrete schemes, high order, WENO, CWENO, monotone fluxes.

AMS(MOS) subject classification. Primary 65M06; secondary 35L99.

\section{Introduction}

We are interested in approximating solutions of multi-dimensional Hamilton-Jacobi (HJ) equations of the form

$$
\phi_{t}+H(\nabla \phi)=0, \quad \vec{x}=\left(x_{1}, \ldots x_{d}\right) \in \mathbb{R}^{d},
$$

where $\phi=\phi(\vec{x}, t)$, and the Hamiltonian, $H$, depends on $\nabla \phi$ and possibly on $x$ and $t$. Solutions of (1.1) develop discontinuous derivatives even for smooth initial data. This

* Program in Scientific Computing/Computational Mathematics, Stanford University and the NASA Advanced Supercomputing Division, NASA Ames Research Center, Moffett Field, CA 94035-1000: bryson@nas.nasa.gov

tDepartment of dlevy@math.stanford.edu 
loss of regularity presents difficulties both in the analysis of these equations as well as in numerically approximating their solutions. Significant advances in the theoretical understanding of the HJ equations were achieved in the last two decades. Most notable is the introduction of the so-called "viscosity solution" which provides a consistent definition of a weak solution of (1.1) past the time where the discontinuities develop. See $[2,7,8,9,10,14,25,26]$ and the references therein.

In spite of the large number of applications for HJ equations, there has been very little activity in numerically approximating their solutions. This is surprising in particular given the equivalence between the HJ equations and hyperbolic conservation laws, and the flourishing field of numerical methods for conservation laws. Converging first order methods for the HJ equations were introduced by Souganidis in [33]. High order upwind methods were introduced by Osher, Sethian and Shu in [31,32]. The schemes in $[31,32]$ were based on an "essentially non-oscillatory" (ENO) reconstruction by Harten [13] and a monotone numerical flux. A more compact upwind scheme which is based on a weighted ENO (WENO) reconstruction is due to Jiang and Peng [15]. WENO reconstructions were originally introduced in the context of numerical schemes for hyperbolic conservation laws in $[16,29]$. They increase the order of accuracy by using wider stencils in smooth regions while automatically switching into one-sided stencils in regions that include singularities. All these reconstructions include nonlinear limiters in order to control the spurious oscillations that might develop in the solution. For extensions to unstructured grids see $[1,34]$.

A class of Godunov-type approximations for $\mathrm{HJ}$ equations was recently introduced by $\operatorname{Lin}$ and Tadmor in $[27,28]$. Their first- and second-order central schemes were based on the first-order Lax-Friedrichs scheme [11] and the second-order NessyahuTadmor scheme [30] for approximating solutions of hyperbolic conservation laws. Central schemes incorporate internal averaging over discontinuities and hence they do not require Riemann solvers. Moreover, systems can be solved without a characteristic decomposition, and this makes central schemes simple, robust, and particularly suitable for treating complex geometries. We developed in [4] an efficient version of the central schemes of $[27,28]$ for multi-dimensional HJ equations. Our first- and second-order, non-oscillatory, non-staggered schemes were designed to scale well with an increasing dimension. Efficiency was obtained by carefully choosing the location of the evolution points and by using a one-dimensional projection step. In $[5,6]$ we introduced thirdand fifth-order fully-discrete central schemes, which were the first central schemes for HJ equations of accuracy greater than two. High-order accuracy was obtained using a suitable high-order WENO-type reconstruction. We would like to note that ENO and WENO interpolants were already used in central schemes for conservation laws $[3,22,23,24]$.

One way to improve the above schemes $[4,5,6,27,28]$ is to use semi-discrete methods to reduce the numerical dissipation. In principle, one expects to obtain a semidiscrete scheme from a fully-discrete scheme in the limit as $\Delta t \rightarrow 0$. Unfortunately, the fully-discrete schemes in $[4,5,6,27,28]$ blow up in that limit. A different strategy is to consider at every grid point more precise information regarding the local speed of propagation, which can then be used to develop a different class of fully-discrete approximations that do enjoy a semi-discrete limit. An estimate of the local speed of 
propagation at every grid point can then be used to determine new points where the solution is evolved to the next time step. The distance of these evolution points from the original grid points is proportional to the time step $\Delta t$, and hence it is possible to obtain a semi-discrete scheme in the limit $\Delta t \rightarrow 0$. This strategy was first used to develop semi-discrete central schemes for hyperbolic conservation laws: a second-order method was developed by Kurganov and Tadmor in [21], and a third-order method by Kurganov and Levy in [18]. Semi-discrete schemes for HJ equations were then introduced in [20], and further improved in [19] by utilizing a more accurate estimate of the local speed of propagation, hence reducing numerical dissipation. We would like to stress that both schemes $[19,20]$ are only second-order accurate.

In this paper we present fifth-order, semi-discrete, Godunov-type, central schemes for HJ equations. These are the first high-order semi-discrete central schemes for $\mathrm{HJ}$ equations. ${ }^{1}$ These schemes are generated by considering a general formulation of semidiscrete schemes along the lines of $[19,20]$, and augmenting it with an appropriate high-order WENO-type reconstruction.

The structure of this paper is as follows. In Section 2 we develop a one-dimensional fifth-order semi-discrete scheme. In the semi-discrete limit, $\Delta t \rightarrow 0$, the fifth-order WENO interpolant we obtain turns to be identical to the one used in upwind methods [15]. The flux we use is the Kurganov-Noelle-Petrova flux [19], or a variant of the simpler Kurganov-Tadmor flux [20]. We state a theorem establishing the monotonicity of these fluxes, the proof of which is left to an appendix. We observe that for the one-dimensional linear advection, our method boils down to an upwind scheme with a Lax-Friedrichs flux. In Section 3 we generalize the method to an arbitrary number of space dimensions, writing out the schemes explicitly for two and three dimensions in Section 3.2. We conclude in Section 4 with several numerical examples in one, two and three space dimensions that confirm the expected order of accuracy as well as the high-resolution nature of our scheme. We compare the results of these numerical tests with our fully-discrete fifth-order scheme [6] and with the scheme of Jiang and Peng [15]. Our numerical results show that the new method we present in this paper has stability properties that are equivalent to those of [15]. The relative $L^{1}$ errors we obtain in all our simulations are consistently smaller than those in [15], in some cases as much as an order of magnitude smaller.

Acknowledgment: The work of D. Levy was supported in part by the National Science Foundation under Career Grant No. DMS-0133511.

\section{A One-Dimensional Scheme}

\subsection{Semi-Discrete Central Schemes for HJ Equations}

Consider the one-dimensional HJ equation of the form

$$
\phi_{t}(x, t)+H\left(\phi_{x}\right)=0, \quad x \in \mathbb{R} .
$$

\footnotetext{
${ }^{1}$ high-order is assumed to be an order greater than two.
} 


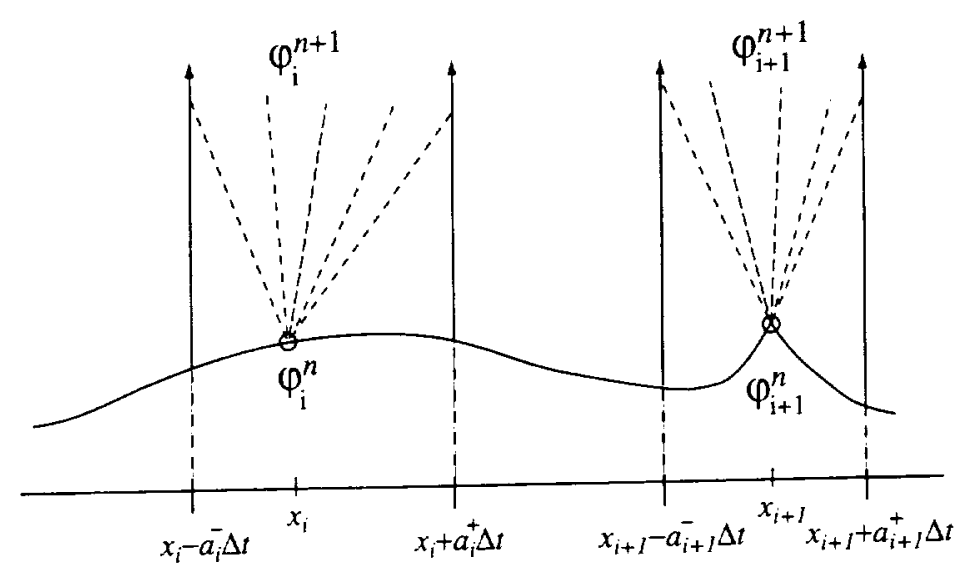

Figure 2.1: The regions considered in Godunov-type central schemes. The solution is evolved at $x_{i} \pm a_{i}^{ \pm} \Delta t$. The solution $\varphi_{i}^{n+1}$ is obtained by averaging $\varphi\left(x_{i} \pm a_{i}^{ \pm} \Delta t, t^{n+1}\right)$.

We are interested in approximating solutions of (2.1) subject to the initial data $\phi(x, t=$ $0)=\phi_{0}(x)$. We briefly review the construction of semi-discrete central schemes for (2.1) presented in [19] (see also [20]). For simplicity we assume a uniform grid grid in space and time with mesh spacings, $\Delta x$ and $\Delta t$, respectively. Denote the grid points by $x_{i}=i \Delta x, t^{n}=n \Delta t$. Let $\varphi_{i}^{n}$ denote the approximate value of $\phi\left(x_{i}, t^{n}\right)$, and at a fixed time $t^{n}$ let $\varphi_{i}^{\prime}$ denote the approximate value of the spatial derivative $\phi_{x}\left(x_{i}, t^{n}\right)$. We define the forward and backward differences $\Delta^{+} \varphi_{i}:=\varphi_{i+1}-\varphi_{i}$ and $\Delta^{-} \varphi_{i}:=\varphi_{i}-\varphi_{i-1}$.

Assume that the approximate solution at time $t^{n}, \varphi_{i}^{n}$ is given, and that a continuous piecewise-polynomial interpolant $\tilde{\varphi}\left(x, t^{n}\right)$ was reconstructed from $\varphi_{i}^{n}$. The construction of $\tilde{\varphi}\left(x, t^{n}\right)$ will be addressed below. At every grid point $x_{i}$ we then estimate the maximal speed of propagation to left, $a_{i}^{+}$, and to the right, $a_{i}^{-}$. For a convex Hamiltonian, these one-sided local speeds of propagation are estimated by

$$
a_{i}^{+}=\max \left\{H^{\prime}\left(\varphi_{i}^{\prime-}\right), H^{\prime}\left(\varphi_{i}^{\prime}\right), 0\right\}, \quad a_{i}^{-}=\left|\min \left\{H^{\prime}\left(\varphi_{i}^{\prime-}\right), H^{\prime}\left(\varphi_{i}^{\prime+}\right), 0\right\}\right| .
$$

Here, $\varphi_{i}^{\prime \pm}$ are the one-sided derivatives, defined as

$$
\varphi_{i}^{\prime \pm}:=\lim _{\Delta t \rightarrow 0} \tilde{\varphi}_{x}\left(x_{i} \pm a_{i}^{ \pm} \Delta t, t^{n}\right) .
$$

Remark. Our sign convention in the definition of $a_{i}^{ \pm}$in (2.2) differs from [19]. This choice of signs simplifies the derivation of the scheme in the multi-dimensional setup.

We evolve $\tilde{\varphi}$ according to (2.1) at the evolution points $x_{i} \pm a_{i}^{ \pm} \Delta t$. The time step, $\Delta t$, is chosen so that the reconstruction is smooth at these points (see Figure 2.1). A Taylor expansion in time of $\tilde{\varphi}\left(x_{i} \pm a_{i}^{ \pm} \Delta t, t^{n+1}\right)$ results with

$$
\tilde{\varphi}\left(x_{i} \pm a_{i}^{ \pm} \Delta t, t^{n+1}\right)=\tilde{\varphi}\left(x_{i} \pm a_{i}^{ \pm} \Delta t, t^{n}\right)-\Delta t H\left(\tilde{\varphi}_{x}\left(x_{i} \pm a_{i}^{ \pm} \Delta t, t^{n}\right)\right)+O\left(\Delta t^{2}\right)
$$

A weighted average is then used to re-project $\tilde{\varphi}\left(x_{i} \pm a_{i}^{ \pm} \Delta t, t^{n+1}\right)$ onto the original grid point $x_{i}$,

$$
\varphi_{i}^{n+1}=\frac{a_{i}^{+}}{a_{i}^{+}+a_{i}^{-}} \tilde{\varphi}\left(x_{i}-a_{i}^{-} \Delta t, t^{n+1}\right)+\frac{a_{i}^{-}}{a_{i}^{+}+a_{i}^{-}} \tilde{\varphi}\left(x_{i}+a_{i}^{+} \Delta t, t^{n+1}\right) .
$$


A fully discrete central scheme is finally obtained by substituting (2.3) into (2.4)

$$
\begin{aligned}
\varphi_{i}^{n+1}= & \frac{a_{i}^{+}}{a_{i}^{+}+a_{i}^{-}}\left(\tilde{\varphi}\left(x_{i}-a_{i}^{-} \Delta t, t^{n}\right)-\Delta t H\left(\tilde{\varphi}_{x}\left(x_{i}-a_{i}^{-} \Delta t, t^{n}\right)\right)\right) \\
& +\frac{a_{i}^{-}}{a_{i}^{+}+a_{i}^{-}}\left(\tilde{\varphi}\left(x_{i}+a_{i}^{+} \Delta t, t^{n}\right)-\Delta t H\left(\tilde{\varphi}_{x}\left(x_{i}+a_{i}^{+} \Delta t, t^{n}\right)\right)\right) .
\end{aligned}
$$

Utilizing the Taylor expansion $\tilde{\varphi}\left(x_{i} \pm a_{i}^{ \pm} \Delta t, t^{n}\right)=\tilde{\varphi}\left(x_{i}, t^{n}\right) \pm a_{i}^{ \pm} \Delta t \tilde{\varphi}_{x}^{ \pm}$, and assuming that $\tilde{\varphi}$ satisfies the interpolation requirements $\varphi_{i}^{n}=\bar{\varphi}\left(x_{i}, t^{n}\right)$, equation (2.5) can be rewritten as

$$
\begin{aligned}
\varphi_{i}^{n+1} & =\varphi_{i}^{n}+\Delta t \frac{a_{i}^{+} a_{i}^{-}}{a_{i}^{+}+a_{i}^{-}}\left[\tilde{\varphi}_{x}^{+}-\tilde{\varphi}_{x}^{-}\right] \\
& -\frac{\Delta t}{a_{i}^{+}+a_{i}^{-}}\left[a_{i}^{-} H\left(\bar{\varphi}_{x}\left(x_{i}+a_{i}^{+} \Delta t, t^{n}\right)\right)+a_{i}^{+} H\left(\tilde{\varphi}_{x}\left(x_{i}-a_{i}^{-} \Delta t, t^{n}\right)\right)\right] .
\end{aligned}
$$

Here $\tilde{\varphi}_{x}^{ \pm}$denotes the one-sided reconstruction of the derivative at $x_{i}$. A Godunov-type semi-discrete method for approximating the solution of $(2.1)$ is obtained by taking the limit $\Delta t \rightarrow 0$ in $(2.6)$ (see $[19$, Eq. (3.44)])

$$
\frac{d}{d t} \varphi_{i}(t)=-\frac{1}{a_{i}^{+}+a_{i}^{-}}\left[a_{i}^{-} H\left(\varphi_{i}^{\prime+}\right)+a_{i}^{+} H\left(\varphi_{i}^{\prime}\right)\right]+\frac{a_{i}^{+} a_{i}^{-}}{a_{i}^{+}+a_{i}^{-}}\left(\varphi_{i}^{\prime+}-\varphi_{i}^{\prime-}\right)
$$

Even though the flux on the right hand side of 2.7 was originally presented in [19], Kurganov et. al. did not investigate its properties. We now state a theorem establishing the monotonicity of this flux. The proof is left to the appendix.

Theorem 2.1 Assume that $H \in C^{2}$ is convex. Then

$$
H^{K N P}\left(u^{+}, u^{-}\right)=\frac{1}{a^{+}+a^{-}}\left[a^{-} H\left(u^{+}\right)+a^{+} H\left(u^{-}\right)\right]-\frac{a^{+} a^{-}}{a^{+}+a^{-}}\left(u^{+}-u^{-}\right) \text {, }
$$

is a monotone flux, that is $H^{K N P}$ is a non-increasing function of $u^{+}$and a nondecreasing function of $u^{-}$.

Remarks.

1. The derivation of the semi-discrete scheme (2.7) does not depend on choice of interpolants $\tilde{\varphi}$, so long as they are smooth at $x_{i} \pm a_{i}^{ \pm} \Delta t$ during the time interval $\left[t^{n}, t^{n+1}\right]$. The spatial order of accuracy of the scheme is determined by the accuracy of the reconstruction of $\tilde{\varphi}$ as well as on the accuracy of the ODE solver used to solve (2.7). A suitable high-order reconstruction will be presented in Section 2.2 below. To be precise, the scheme (2.7) does not require the construction of the interpolant $\tilde{\varphi}$. All that is required is a reconstruction of the point-values of the derivatives $\tilde{\varphi}^{\prime \pm}$. 


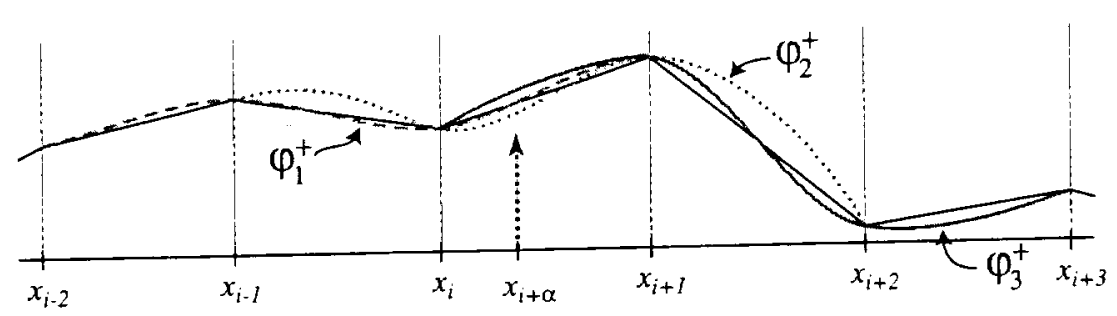

Figure 2.2: The three interpolants used for the fifth-order reconstruction $\varphi_{i}^{\prime+}$. In this example, because of the large gradient between $x_{i+1}$ and $x_{i+2}$, the interpolant $\varphi_{-}$will have the strongest contribution to the CWENO reconstruction at $x_{i+\tau}$.

2. In order to economize on storage space, and sometimes also reduce the computations, it is possible to replace $a_{i}^{+}$and $a_{i}^{-}$with $a_{i}=\max \left\{\left|H^{\prime}\left(\varphi_{i}^{\prime-}\right)\right|,\left|H^{\prime}\left(\varphi_{i}^{\prime+}\right)\right|\right\}$. In this case, (2.7) becomes

$$
\frac{d}{d t} \varphi_{i}(t)=-\frac{1}{2}\left[H\left(\varphi_{i}^{\prime+}\right)+H\left(\varphi_{i}^{\prime-}\right)\right]+\frac{a_{i}}{2}\left(\varphi_{i}^{\prime+}-\varphi_{i}^{\prime-}\right)
$$

This simpler formulation was presented by Kurganov and Tadmor in [20, Eq.

(4.10)]. We denote the right hand side of $(2.8)$ by $-H^{K T}\left(\varphi_{i}^{\prime+}, \varphi_{i}^{\prime-}\right)$.

As an immediate consequence of Theorem 2.1, we have

Corollary 2.1 If $H \in C^{2}$ is convex, then $H^{K T}\left(u^{+}, u^{-}\right)$is a monotone flux.

\subsection{A Fifth-Order Scheme}

In order to obtain a fifth-order scheme from the general semi-discrete formulation (2.7), we need a fifth-order approximation of the derivative $\varphi^{\prime}$ and a suitable ODE solver. A central-upwind interpolant at $x_{i}$ starts with a central interpolant defined either on the interval $\left[x_{i}, x_{i+1}\right]$ for a right-biased reconstruction, or $\left[x_{i-1}, x_{i}\right]$ for a left-biased reconstruction. This central interpolant is then evaluated at the location $x_{i+\tau}:=x_{i}+\tau \Delta x$, where $\tau$ is a parameter introduced for notational convenience. For the semi-discrete scheme (2.7) we take $\tau=a_{i}^{+} \Delta t$ for the right-biased interpolant, and $\tau=a_{i}^{-} \Delta t$ for the left-biased interpolant.

For the right-biased interpolant at $x_{i+\tau}\left(\tau=a_{i}^{+} \Delta t\right)$, we use three cubic interpolants $\varphi_{k, i+\tau}^{+}, k=1,2,3$, defined on the stencil $\left\{x_{i-3+k}, \ldots, x_{i+k}\right\}$. Here

$$
\begin{aligned}
\varphi_{1, i+\tau}^{\prime+}= & \frac{1}{6 \Delta x}\left[\left(1-3 \tau^{2}\right) \varphi_{i-2}+3\left(-2+2 \tau+3 \tau^{2}\right) \varphi_{i-1}\right. \\
& \left.+3\left(1-4 \tau-3 \tau^{2}\right) \varphi_{i}+\left(2+6 \tau+3 \tau^{2}\right) \varphi_{i+1}\right], \\
\varphi_{2, i+\tau}^{\prime+}= & \frac{1}{6 \Delta x}\left[\left(-2+6 \tau-3 \tau^{2}\right) \varphi_{i-1}+3\left(-1-4 \tau+3 \tau^{2}\right) \varphi_{i}\right. \\
& \left.+3\left(2+2 \tau-3 \tau^{2}\right) \varphi_{i+1}+\left(-1+3 \tau^{2}\right) \varphi_{i+2}\right],
\end{aligned}
$$




$$
\begin{aligned}
\varphi_{3, i+\tau}^{\prime+}= & \frac{1}{6 \Delta x}\left[\left(-11+12 \tau-3 \tau^{2}\right) \varphi_{i}+3\left(6-10 \tau+3 \tau^{2}\right) \varphi_{i+1}\right. \\
& \left.+3\left(-3+8 \tau-3 \tau^{2}\right) \varphi_{i+2}+\left(2-6 \tau+3 \tau^{2}\right) \varphi_{i+3}\right]
\end{aligned}
$$

A straightforward computation shows that $\forall k, \varphi_{k, i+\tau}^{+}=\frac{\partial}{\partial x} \varphi\left(x_{i+\tau}\right)+O\left((\Delta x)^{3}\right)$. Also the following linear combination is a fifth-order approximation of $\varphi_{i}^{\prime+}$

$$
\hat{\varphi}_{i+\tau}^{\prime+}=\sum_{k=1}^{3} c_{k} \varphi_{k, i+\tau}^{\prime+}=\frac{\partial}{\partial x} \varphi\left(x_{i+\tau}\right)+O\left((\Delta x)^{5}\right),
$$

provided that the constants $c_{k}$ are taken as

$$
\begin{aligned}
& c_{1}=\frac{1}{20} \frac{15 \tau^{2}+10 \tau-6-120 \tau^{3}+120 \tau^{4}}{3 \tau^{2}-1} \\
& c_{2}=-\frac{1}{20} \frac{720 \tau^{6}-1080 \tau^{5}+660 \tau^{4}+60 \tau^{3}-81 \tau^{2}-64 \tau+24}{\left(3 \tau^{2}-1\right)\left(2-6 \tau+3 \tau^{2}\right)} \\
& c_{3}=\frac{1}{20} \frac{-15 \tau^{2}+4+120 \tau^{4}}{2-6 \tau+3 \tau^{2}} .
\end{aligned}
$$

In the limit $\tau \rightarrow 0, \varphi_{k, i}^{++}:=\lim _{\tau \rightarrow 0} \varphi_{k, i+\tau}^{\prime+}=\frac{\partial \varphi}{\partial x}\left(x_{i}\right)+O\left((\Delta x)^{3}\right)$, with

$$
\begin{aligned}
\varphi_{1, i}^{\prime+} & =\frac{1}{6 \Delta x}\left(\varphi_{i-2}-6 \varphi_{i-1}+3 \varphi_{i}+2 \varphi_{i+1}\right) \\
\varphi_{2, i}^{\prime+} & =\frac{1}{6 \Delta x}\left(-2 \varphi_{i-1}-3 \varphi_{i}+6 \varphi_{i+1}-\varphi_{i+2}\right) \\
\varphi_{3, i}^{\prime+} & =\frac{1}{6 \Delta x}\left(-11 \varphi_{i}+18 \varphi_{i+1}-9 \varphi_{i+2}+2 \varphi_{i+3}\right)
\end{aligned}
$$

A right-biased fifth-order interpolant at $x_{i}$ is therefore given by

$$
\hat{\varphi}_{i}^{\prime+}=\frac{3}{10} \varphi_{1, i}^{\prime+}+\frac{3}{5} \varphi_{2, i}^{\prime+}+\frac{1}{10} \varphi_{3, i}^{\prime+}=\frac{\partial}{\partial x} \varphi\left(x_{i}\right)+O\left((\Delta x)^{5}\right)
$$

By symmetry, for the left-biased interpolant $\left(\tau=a_{i}^{-} \Delta t\right)$ we use three cubic interpolants $\varphi_{k, i+\tau}^{\prime}, k=1,2,3$, this time defined on the stencil $\left\{x_{i-4+k}, \ldots, x_{i-1+k}\right\}$. In the limit $\tau \rightarrow 0, \varphi_{k, i}^{\prime-}:=\lim _{\tau \rightarrow 0} \varphi_{k, i+\tau}^{\prime-}=\frac{\partial \varphi}{\partial x}\left(x_{i}\right)+O\left((\Delta x)^{3}\right)$, where

$$
\begin{aligned}
\varphi_{1, i}^{\prime-} & =\frac{1}{6 \Delta x}\left(2 \varphi_{i-3}-9 \varphi_{i-2}+18 \varphi_{i-1}-11 \varphi_{i}\right) \\
\varphi_{2, i}^{\prime-} & =\frac{1}{6 \Delta x}\left(-\varphi_{i-2}+6 \varphi_{i-1}-3 \varphi_{i}-2 \varphi_{i+1}\right) \\
\varphi_{3, i}^{\prime-} & =\frac{1}{6 \Delta x}\left(2 \varphi_{i-1}+3 \varphi_{i}-6 \varphi_{i+1}+\varphi_{i+2}\right)
\end{aligned}
$$

In this case

$$
\hat{\varphi}_{i}^{\prime-}=\frac{1}{10} \varphi_{1, i}^{\prime-}+\frac{3}{5} \varphi_{2, i}^{\prime-}+\frac{3}{10} \varphi_{3, i}^{\prime-}=\frac{\partial}{\partial x} \varphi\left(x_{i}\right)+O\left((\Delta x)^{5}\right)
$$


In order to suppress spurious oscillations, the coefficients in $\hat{\varphi}_{i}^{\prime \pm}$ are replaced by nonlinear weights, which are set as to preserve the order of accuracy of the reconstruction in smooth regions while automatically switching to the appropriate stencil in regions that contain discontinuities. To this end we define the convex combination

$$
\varphi_{i}^{\prime \pm}=\sum_{k=1}^{3} w_{k, i}^{ \pm} \varphi_{k, i}^{ \pm}, \quad \sum_{k=1}^{3} w_{k, i}^{ \pm}=1
$$

In smooth regions $w_{1, i}^{+} \approx w_{3, i}^{-} \approx c_{1}^{+}=c_{3}^{-}=3 / 10, w_{3, i}^{+} \approx w_{1, i}^{-} \approx c_{3}^{+}=c_{1}^{-}=1 / 10$ and $w_{2, i}^{ \pm} \approx c_{2}^{ \pm}=3 / 5$ so the order is of the order $O\left((\Delta x)^{5}\right)$. When the stencil supporting $\varphi_{k, i}^{ \pm}$ contains a discontinuity, the weight of the more oscillatory polynomial should vanish. Following $[16,29]$, these requirements are met by setting

$$
w_{k, i}^{ \pm}=\frac{\omega_{k, i}^{ \pm}}{\sum_{l} \omega_{l, i}^{ \pm}}, \quad \omega_{k, i}^{ \pm}=\frac{c_{k}^{ \pm}}{\left(\epsilon+S_{k, i}^{ \pm}\right)^{p}}
$$

where $k, l \in\{1,2,3\}$. We choose $\epsilon$ as $10^{-6}$ to prevent the denominator in (2.13) from vanishing, and set $p=2$ (see [16]). The smoothness measures $S_{k, i}^{ \pm}$should be large when $\varphi$ is nearly singular. Following [16], we take $S_{k, i}^{ \pm}$to be the sum of the $L^{2}$-norms of the derivatives on the stencil supporting $\varphi_{k, i}^{\prime \pm}$. If we approximate the first derivative at $x_{i}$ by $\Delta^{+} \varphi_{i} / \Delta x$, the second derivative by $\Delta^{+} \Delta^{-} \varphi_{i \pm \tau} /(\Delta x)^{2}$, and define the smoothness measure

$$
S_{i}[r, s]=\Delta x \sum_{j=r}^{s}\left(\frac{1}{\Delta x} \Delta^{+} \varphi_{i+j}\right)^{2}+\Delta x \sum_{j=r+1}^{s}\left(\frac{1}{\Delta x^{2}} \Delta^{+} \Delta^{-} \varphi_{i+j}\right)^{2},
$$

then for the right-biased interpolant we have $S_{1, i}^{+}=S_{i}[-2,0], S_{2, i}^{+}=S_{i}[-1,1]$ and $S_{3, i}^{+}=S_{i}[0,2]$. For left-biased interpolant we have $S_{1, i}^{-}=S_{i}[-3,-1], S_{2, i}^{-}=S_{i}[-2,0]$ and $S_{3, i}^{-}=S_{i}[-1,1]$. We use the notation

$$
\varphi^{\prime \pm}=\text { reconstruct }-\varphi^{\prime}\left( \pm, \varphi^{n}\right)
$$

to denote the computation of the array $\left\{\varphi_{i}^{\prime \pm}\right\}$ for all $i$ from data $\varphi^{n}$ at time $t^{n}$, as given by (2.12).

The following algorithm summarizes our fifth-order semi-discrete algorithm for approximatinng the solution of $(2.7)$. The time integration is performed with a fourth-order strong stability preserving (SSP) Runge-Kutta scheme [12].

Algorithm 2.1 Let $F\left(\varphi_{i}^{\prime-}, \varphi_{i}^{\prime+}\right)$ denote the right hand side of (2.7). Then at each grid node $i$,

$$
\begin{aligned}
& \varphi_{0}^{\prime-}=\text { reconstruct }_{-} \varphi^{\prime}\left(-, \varphi^{n}\right), \quad \varphi_{0}^{\prime+}={\text { reconstruct }-\varphi^{\prime}}^{\prime}\left(+, \varphi^{n}\right) \\
& \varphi^{(1)}=\varphi^{n}+\frac{1}{2} \Delta t F\left(\varphi_{0}^{\prime-}, \varphi_{0}^{\prime+}\right) \\
& \left.\varphi_{1}^{\prime-}={\text { reconstruct }-\varphi^{\prime}}^{\prime}-, \varphi^{(1)}\right), \quad \varphi_{1}^{\prime+}=\text { reconstruct }_{-} \varphi^{\prime}\left(+, \varphi^{(1)}\right)
\end{aligned}
$$




$$
\begin{aligned}
\varphi^{(2)}= & \frac{649}{1600} \varphi^{n}-\frac{10890423}{25193600} \Delta t F\left(\varphi_{0}^{\prime-}, \varphi_{0}^{\prime+}\right)+\frac{951}{1600} \varphi^{(1)}+\frac{5000}{7873} \Delta t F\left(\varphi_{1}^{\prime-}, \varphi_{1}^{\prime+}\right) \\
\varphi_{2}^{\prime-}= & \text { reconstruct_} \varphi^{\prime}\left(-, \varphi^{(2)}\right), \quad \varphi_{2}^{\prime+}=\text { reconstruct_}_{-}\left(+, \varphi^{(2)}\right) \\
\varphi^{(3)}= & \frac{53989}{2500000} \varphi^{n}-\frac{102261}{5000000} \Delta t F\left(\varphi_{0}^{\prime-}, \varphi_{0}^{\prime+}\right)+\frac{4806213}{20000000} \varphi^{(1)} \\
& -\frac{5121}{20000} \Delta t F\left(\varphi_{1}^{\prime-}, \varphi_{1}^{\prime+}\right)+\frac{23619}{32000} \varphi^{(2)}+\frac{7873}{10000} \Delta t F\left(\varphi_{2}^{\prime-}, \varphi_{2}^{\prime+}\right) \\
\varphi_{3}^{\prime-}= & \text { reconstruct_} \varphi^{\prime}\left(-, \varphi^{(3)}\right), \quad \varphi_{3}^{\prime+}=\text { reconstruct_-}^{\prime}\left(+, \varphi^{(3)}\right) \\
\varphi^{n+1} & =\frac{1}{5} \varphi^{n}+\frac{1}{10} \Delta t F\left(\varphi_{0}^{\prime-}, \varphi_{0}^{\prime+}\right)+\frac{6127}{30000} \varphi^{(1)}+\frac{1}{6} \Delta t F\left(\varphi_{1}^{\prime-}, \varphi_{1}^{\prime+}\right)+\frac{7873}{30000} \varphi^{(2)} \\
& +\frac{1}{3} \varphi^{(3)}+\frac{1}{6} \Delta t F\left(\varphi_{3}^{\prime-}, \varphi_{3}^{\prime+}\right)
\end{aligned}
$$

\section{Remarks.}

1. The smoothness measures (2.14) are not the same as those used in $[15,16]$. There, a different normalization of the derivatives was used. Our smoothness measures are approximations to the sum of the $L^{2}$-norms of the first and second derivatives of the interpolant on a stencil. In the cases we tested our smoothness measures produced comparable or smaller errors when compared with [15]. We include a comparison between the results obtained with both forms of the smoothness measures in Section 4.1.1.

2. From obvious reasons, the interpolant (2.10) is identical to the one used in the upwind method of [15]. As far as the scheme itself is concerned, there is some degree of similarity between the semi-discrete central scheme and upwind schemes. It is important to note that for linear advection problems they boil down to the same scheme. Indeed, if $H(s)=s$, then $H^{\prime}=1$. Hence $a_{j}^{+}=1$, $a_{j}^{-}=0$ and equation (2.7) becomes

$$
\frac{d}{d t} \varphi_{j}(t)=-\frac{1}{a_{j}^{+}}\left[a_{j}^{+} H\left(\varphi_{i}^{\prime-}\right)\right]=-\varphi_{i}^{\prime-}
$$

Solving (2.16) is equivalent to solving

$$
\frac{d}{d t} \varphi_{j}(t)=-H^{L F}\left(\varphi_{i}^{\prime+}, \varphi_{i}^{\prime-}\right)
$$

with the Lax-Friedrichs flux

$$
H^{L F}\left(\varphi_{i}^{\prime+}, \varphi_{i}^{\prime-}\right)=H\left(\frac{1}{2}\left(\varphi_{i}^{\prime+}+\varphi_{i}^{\prime-}\right)\right)-\frac{1}{2}\left(\varphi_{i}^{\prime+}-\varphi_{i}^{\prime-}\right)=\varphi_{i}^{\prime-}
$$


For this reason the schemes in [19] are called "central-upwind schemes". Even in this case of a linear advection problem, there still are some differences between our scheme (Algorithm 2.1) and the scheme in [15]: the ODE solvers and the smoothness measures are different. For more complicated Hamiltonians the semi-discrete scheme (2.7) is different than the scheme in [15]. A comparison between numerical results obtained with both schemes can be found in Section 4.

3. One can easily create a third-order semi-discrete central scheme from the general one-dimensional formulation (2.7) by using a less accurate ODE solver and a third-order interpolant. Indeed, a third-order version of the right-biased (derivatives of the) interpolants can be written as a combination of two polynomials, $\varphi_{j, i}^{\prime}$ with $j=1,2$, that are constructed on the stencil $\left\{x_{j+i-2}, \ldots, x_{j+i}\right\}$ (compare with (2.9)). A straightforward computation shows that

$$
\begin{aligned}
\varphi_{1, i}^{\prime} & =\lim _{\tau \rightarrow 0} \frac{1}{\Delta x}\left[\left(-\frac{1}{2}+\tau\right) \varphi_{i-1}+(-2 \tau) \varphi_{i}+\left(\frac{1}{2}+\tau\right) \varphi_{i+1}\right] \\
& =\frac{1}{2 \Delta x}\left(\varphi_{i+1}-\varphi_{i-1}\right) \\
\varphi_{2, i}^{\prime} & =\lim _{\tau \rightarrow 0} \frac{1}{\Delta x}\left[\left(-\frac{3}{2}+\tau\right) \varphi_{i}+(2-2 \tau) \varphi_{i+1}+\left(-\frac{1}{2}+\tau\right) \varphi_{i+2}\right] \\
& =\frac{1}{2 \Delta x}\left(-3 \varphi_{i}+4 \varphi_{i+1}-\varphi_{i+2}\right)
\end{aligned}
$$

satisfies $\varphi_{j, i}^{\prime}=\partial \varphi\left(x_{i+\tau}\right) / \partial x+O\left((\Delta x)^{2}\right)$ for $\tau \neq 0$ and $j=1,2$. The combination

$$
\varphi_{c, i}^{\prime}=\lim _{\tau \rightarrow 0}\left[-\frac{1}{3} \frac{2-6 \tau+3 \tau^{2}}{-1+2 \tau} \varphi_{1, i}^{\prime}+\frac{1}{3} \frac{-1+3 \tau^{2}}{-1+2 \tau} \varphi_{2, i}^{\prime}\right]=\frac{2}{3} \varphi_{1, i}^{\prime}+\frac{1}{3} \varphi_{2, i}^{\prime}
$$

satisfies $\varphi_{c, i}^{\prime}=\partial \varphi\left(x_{i+\tau}\right) / \partial x+O\left((\Delta x)^{3}\right)$. The left-biased interpolants can be easily derived by symmetry considerations.

\section{Multi-Dimensional Schemes}

\subsection{A General Multi-Dimensional Scheme}

Consider the $d$-dimensional $\mathrm{HJ}$ equation of the form

$$
\phi_{\mathrm{t}}+H(\nabla \phi)=0, \quad \vec{x}=\left(x^{(1)}, \ldots, x^{(d)}\right) \in \mathbb{R}^{d}
$$

subject to the initial data $\phi(\vec{x}, t=0)=\phi_{0}(\vec{x})$.

For simplicity we assume a uniform grid in space $\Delta x^{(1)}=\cdots=\Delta x^{(d)}=\Delta x$. We set $\vec{\alpha}=\left(\alpha_{1}, \alpha_{2}, \ldots, \alpha_{d}\right) \in \mathbb{Z}^{d}$, and let $\vec{x}_{\alpha}=\Delta x \vec{\alpha}$, such that the $k$-th coordinate of $\vec{x}_{\alpha}$ equals $x_{\alpha}^{(k)}=\Delta x \alpha^{(k)}, \forall 1 \leq k \leq d$. For example, in the conventional three-dimensional notation with indices $i, j$ and $k$ and components $(x, y, z), \vec{\alpha}=(i, j, k)$ and $\vec{x}_{\alpha}=\left(x_{i}, y_{j}, z_{k}\right)$. 
Similarly to the one-dimensional setup, $\varphi_{\alpha}^{n}$ will denote the approximation of $\phi\left(x_{\alpha}, t^{n}\right)$, and for a fixed time $t^{n}, \nabla \varphi_{\alpha}$ will denote the approximation of $\nabla \phi$ at $x_{\alpha}$.

Given $\vec{x}_{\alpha}$, we define the volume $C_{\alpha}=\bigotimes_{k=1}^{d}\left[x_{\alpha}^{(k)}-\frac{\Delta x}{2}, x_{\alpha}^{(k)}+\frac{\Delta x}{2}\right]$, and estimate the local speeds of propagation $\vec{a}_{\alpha}^{ \pm}$. For example, for a convex Hamiltonian these speeds in the coordinate direction $k$ are given by

$$
a_{\alpha}^{(k)+}=\max _{C_{\alpha}}\left\{\frac{\partial H}{\partial x^{(k)}}\left(\nabla \varphi_{\alpha}\right), 0\right\}, \quad a_{\alpha}^{(k)-}=\left|\min _{C_{\alpha}}\left\{\frac{\partial H}{\partial x^{(k)}}\left(\nabla \varphi_{\alpha}\right), 0\right\}\right| .
$$

Let $\vec{\rho}=\left(\rho^{(1)}, \ldots, \rho^{(d)}\right)$ denote the multi-index with components $\rho^{(k)} \in\{+,-\}, \forall k$. We also denote the index opposite to $\vec{\rho}$ by $\bar{\rho}$, i.e. $\bar{\rho}=-\vec{\rho}=\left(-\rho^{(1)}, \ldots,-\rho^{(d)}\right)$, assuming the standard algebraic operations between elements in $\mathbb{Z}$. For any given $\vec{\rho}$ we define a vector that encodes the maximum estimated speed of propagation in all coordinate directions at $\vec{x}_{\alpha}$ as

$$
\vec{v}_{\alpha}^{\rho}=\left(\rho^{(1)} a_{\alpha}^{(1) \rho^{(1)}}, \ldots, \rho^{(d)} a_{\alpha}^{(d) \rho^{(d)}}\right) .
$$

We then denote by $\vec{x}_{\alpha+\rho}$ the position $\vec{x}_{\alpha}+\vec{v}_{\alpha}^{\rho} \Delta t$, and denote the approximation of $\phi$ at $\vec{x}_{\alpha+\rho}$ by $\tilde{\varphi}_{\alpha+\rho}$.

For example, if $d=3$ and $\vec{\rho}=(+,-,+)$, then $\vec{v}_{\alpha}^{\rho}=\left(a_{\alpha \alpha}^{(1)+},-a_{\alpha}^{(2)-}, a_{\alpha}^{(3)+}\right)$ and $\vec{v}_{\alpha}^{\vec{\rho}}=$ $\left(-a_{\alpha}^{(1)-}, a_{\alpha}^{(2)+},-a_{\alpha}^{(3)-}\right)$. In this case

$$
\tilde{\varphi}_{\alpha+\rho}=\tilde{\varphi}\left(x_{\alpha}^{(1)}+a_{\alpha}^{(1)+} \Delta t, x_{\alpha}^{(2)}-a_{\alpha}^{(2)-} \Delta t, x_{\alpha}^{(3)}+a_{\alpha}^{(3)+} \Delta t\right) .
$$

Similarly to the one-dimensional case, we assume that the approximate solution at time $t^{n}, \varphi_{\alpha}^{n}$ is given, and that a continuous piecewise-polynomial interpolant $\tilde{\varphi}\left(\vec{x}, t^{n}\right)$ was reconstructed from $\varphi_{\alpha}^{n}$. The construction of $\tilde{\varphi}\left(\vec{x}, t^{n}\right)$ will be addressed below. The interpolant $\tilde{\varphi}\left(\vec{x}, t^{n}\right)$ is then evolved to the next time step, $t^{n+1}$, at the points $\vec{x}_{\alpha+\rho}$, which are located away from the propagating discontinuities assuming that the time step $\Delta t$ is sufficiently small. According to (3.1), to first order in time, this evolution is given by the Taylor expansion

$$
\tilde{\varphi}\left(\vec{x}_{\alpha+\rho}, t^{n+1}\right)=\tilde{\varphi}\left(\vec{x}_{\alpha+\rho}, t^{n}\right)-\Delta t H\left(\nabla \tilde{\varphi}\left(\vec{x}_{\alpha+\rho}, t^{n}\right)\right)+O\left(\Delta t^{2}\right)
$$

where $\nabla \tilde{\varphi}\left(\vec{x}_{\alpha+\rho}, t^{n}\right)$ is an approximation of the derivative $\nabla \phi$ at $\vec{x}_{\alpha+\rho}$.

A fully discrete central scheme can then be constructed by computing a weighted average of the evolved solution $\tilde{\varphi}\left(\vec{x}_{\alpha+\rho}, t^{n+1}\right)$ for all values of $\vec{\rho}$ (compare with the onedimensional case (2.4)). The volume of the $d$-cube enclosed by $\vec{x}_{\alpha+\rho}$ for all values of $\vec{\rho}$ divided by $\Delta t$ is

$$
V_{\alpha}=\prod_{k=1}^{d}\left(a_{\alpha}^{(k)+}+a_{\alpha}^{(k)-}\right)
$$

For a given $\vec{\rho}$, the volume enclosed by the corners $\vec{x}_{\alpha+\rho}$ and $\vec{x}_{\alpha}$ divided by $\Delta t$ is given by the product of the components of $\vec{v}_{\alpha}^{\rho}$

$$
\left|\vec{v}_{\alpha}^{\rho}\right|=\prod_{k=1}^{d} a_{\alpha}^{(k) \rho^{(k)}}
$$




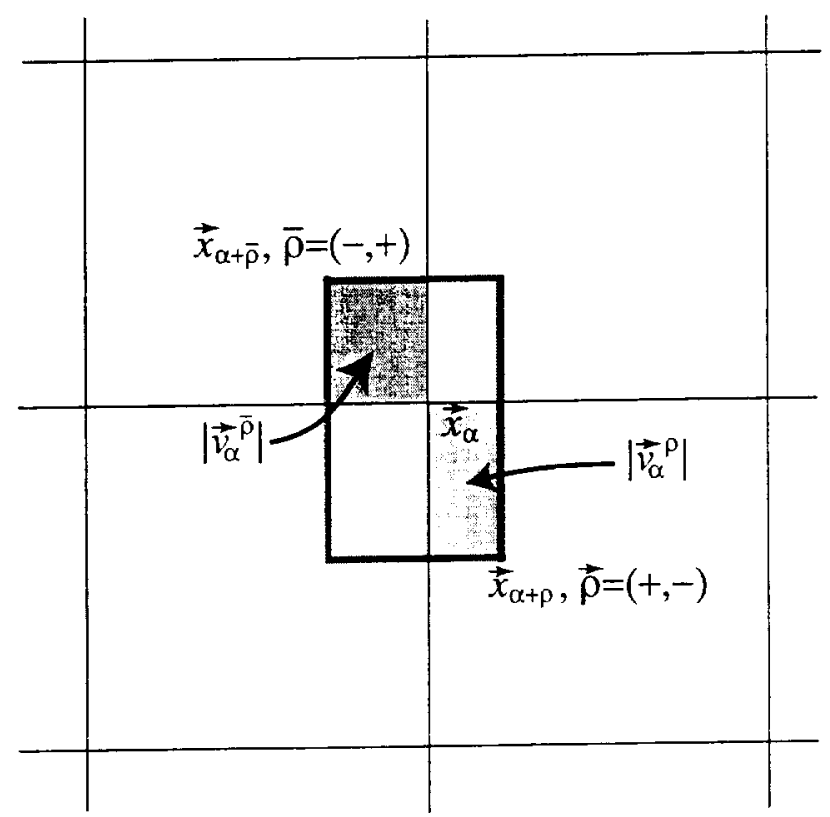

Figure 3.1: A two-dimensional example of the objects associated with the location $\vec{x}_{\alpha+\rho}$ for $\vec{\rho}=(+,-)$. The complement location $\vec{x}_{\alpha+\bar{\rho}}$ is shown, as well as the volumes $\left|\vec{v}_{\alpha}^{\rho}\right|$ and $\left|\vec{v}_{\alpha}^{\bar{\rho}}\right|$. The thick rectangle encloses the volume $V_{\alpha}$.

Clearly, $\sum_{\rho}\left|\vec{v}_{\alpha}^{\rho}\right|=V_{\alpha}$. See Figure 3.1 for a sketch of the two-dimensional setup. An approximation of the solution $\varphi_{\alpha}^{n+1}$ is then obtained by averaging over all $\tilde{\varphi}\left(\vec{x}_{\alpha+\rho}, t^{n+1}\right)$. Each term corresponding to a particular $\vec{\rho}$ is weighted by the diagonally opposite volume $\left|\vec{v}_{\alpha}^{\bar{\rho}}\right|$, divided by $V_{\alpha}$. Hence

$$
\begin{aligned}
\varphi_{\alpha}^{n+1} & =\frac{1}{V_{\alpha}} \sum_{\rho}\left|\vec{v}_{\alpha}^{\bar{\rho}}\right| \tilde{\varphi}\left(\vec{x}_{\alpha+\rho}, t^{n+1}\right) \\
& =\frac{1}{V_{\alpha}} \sum_{\rho}\left|\vec{v}_{\alpha}^{\bar{\rho}}\right|\left[\tilde{\varphi}\left(\vec{x}_{\alpha+\rho}, t^{n}\right)-\Delta t H\left(\nabla \tilde{\varphi}\left(\vec{x}_{\alpha+\rho}, t^{n}\right)\right)\right]
\end{aligned}
$$

We now use a Taylor expansion in space

$$
\tilde{\varphi}\left(\vec{x}_{\alpha+\rho}, t^{n}\right)=\tilde{\varphi}\left(\vec{x}_{\alpha}, t^{n}\right)+\Delta t \vec{v}_{\alpha}^{\rho} \cdot \nabla \tilde{\varphi}\left(\vec{x}_{\alpha+\rho}, t^{n}\right)+O\left(\Delta t^{2}\right)
$$

where $\nabla \tilde{\varphi}\left(\vec{x}_{\alpha+\rho}, t^{n}\right)$ is the evaluation of the gradient at $\vec{x}_{\alpha}$ associated with the reconstruction at $\vec{x}_{\alpha+\rho}$ in the appropriate volume. Hence (3.4) can be written as

$$
\varphi_{\alpha}^{n+1}=\varphi_{\alpha}^{n}+\frac{\Delta t}{V_{\alpha}} \sum_{\rho}\left|\vec{v}_{\alpha}^{\bar{\rho}}\right|\left[\vec{v}_{\alpha}^{\rho} \cdot \nabla \tilde{\varphi}\left(\vec{x}_{\alpha+\rho}, t^{n}\right)-H\left(\nabla \tilde{\varphi}\left(\vec{x}_{\alpha+\rho}, t^{n}\right)\right)\right] .
$$

In the limit $\Delta t \rightarrow 0$ we obtain our first form of the semi-discrete d-dimensional scheme:

$$
\frac{d}{d t} \varphi_{\alpha}(t)=\frac{1}{V_{\alpha}(t)} \sum_{\rho}\left|\vec{v}_{\alpha}^{\bar{\rho}}(t)\right|\left[\vec{v}_{\alpha}^{\rho} \cdot \nabla \tilde{\varphi}_{\alpha+\rho}(t)-H\left(\nabla \tilde{\varphi}_{\alpha+\rho}(t)\right)\right]
$$




$$
=\frac{1}{\prod_{k=1}^{d}\left(a_{\alpha}^{(k)+}+a_{\alpha}^{(k)-}\right)} \sum_{\rho}\left(\prod_{k=1}^{d} a_{\alpha}^{(k) \bar{\rho}^{(k)}}\right)\left[\vec{v}_{\alpha}^{\rho} \cdot \nabla \tilde{\varphi}_{\alpha+\rho}(t)-H\left(\nabla \tilde{\varphi}_{\alpha+\rho}(t)\right)\right] .
$$

To obtain a simpler formula, we let, for $\rho^{(k)}= \pm, \varphi_{x^{(k)}}^{ \pm}=\lim _{\Delta t \rightarrow 0} \partial \tilde{\varphi}\left(\vec{x}_{\alpha+\rho}\right) / \partial x^{(k)}$, the $k$-th component of $\nabla \tilde{\varphi}\left(\vec{x}_{\alpha+\rho}\right)$. Such a limit makes sense assuming that the reconstruction of the derivatives is done direction-by-direction. Then the first sum on the RHS of (3.5) becomes

$$
\begin{aligned}
& \frac{1}{V_{\alpha}} \sum_{k=1}^{d} \sum_{\rho} \prod_{j=1}^{d} a_{\alpha}^{(j) \bar{\rho}^{(j)}} \rho^{(k)} a_{\alpha}^{(k) \rho^{(k)}} \tilde{\varphi}_{x^{(k)}}\left(\vec{x}_{\alpha+\rho}\right) \\
& \quad=\frac{1}{V_{\alpha}} \sum_{k=1}^{d} \sum_{\rho} \prod_{j=1}^{d} a_{\alpha}^{(j) \bar{\rho}^{(j)}}\left(a_{\alpha}^{(k)+} \varphi_{x^{(k)}}^{+}-a_{\alpha}^{(k)-} \varphi_{x^{(k)}}^{-}\right) \\
& \quad=\frac{1}{V_{\alpha}} \sum_{k=1}^{d} a_{\alpha}^{(k)+} a_{\alpha}^{(k)-}\left(\varphi_{x^{(k)}}^{+}-\varphi_{x^{(k)}}^{-}\right) \sum_{\rho} \prod_{j=1 \neq k}^{d} a_{\alpha}^{(j) \bar{\rho}^{(j)}} \\
& \quad=\sum_{k=1}^{d} \frac{a_{\alpha}^{(k)+} a_{\alpha}^{(k)-}}{a_{\alpha}^{(k)+}+a_{\alpha}^{(k)-}}\left(\varphi_{x^{(k)}}^{+}-\varphi_{x^{(k)}}^{-}\right) \frac{\sum_{\rho} \prod_{j=1 \neq k}^{d} a_{\alpha}^{(j) \bar{\rho}(j)}}{\prod_{j=1 \neq k}^{d}\left(a_{\alpha}^{(j)+}+a_{\alpha}^{(j)-}\right)} \\
& \quad=\sum_{k=1}^{d} \frac{a_{\alpha}^{(k)+} a_{\alpha}^{(k)-}}{a_{\alpha}^{(k)+}+a_{\alpha}^{(k)-}}\left(\varphi_{x^{(k)}}^{+}-\varphi_{x^{(k)}}^{-}\right)
\end{aligned}
$$

This gives the semi-discrete d-dimensional scheme

$$
\frac{d}{d t} \varphi_{\alpha}(t)=-\frac{1}{V_{\alpha}} \sum_{\rho}\left|\vec{v}_{\alpha}^{\bar{\rho}}\right| H\left(\nabla \tilde{\varphi}_{\alpha+\rho}^{n}\right)+\sum_{k=1}^{d} \frac{a_{\alpha}^{(k)+} a_{\alpha}^{(k)-}}{a_{\alpha}^{(k)+}+a_{\alpha}^{(k)-}}\left(\varphi_{x^{(k)}}^{+}-\varphi_{x^{(k)}}^{-}\right) .
$$

Remarks.

1. The $d$-dimensional semi-discrete scheme (3.5) is valid for any reconstruction of $\nabla \varphi$, including reconstructions defined on $d$-dimensional stencils (for two-dimensional examples see [6]). In contrast, (3.6) is valid only for dimension-by-dimension reconstructions such as those described in Section 3.3 below. These dimension-by-dimension reconstructions are natural in the semi-discrete setting, as they significantly simplify the form of the scheme.

2. As in the one-dimensional case, (3.5) and (3.6) are independent of the order of the reconstruction. First- and second order-reconstructions can be found, e.g., in [19]. In Section 3.3 we develop a fifth-order dimension-by-dimension reconstruction following the one-dimensional reconstruction of Section 2.2 .

3. Proof of the monotonicity of the flux approximation in (3.6) can be obtained via the method of proof of theorem 2.1 applied to each component. This becomes particularly transparent when (3.6) is written out as in Section 3.2 below. Such a proof cannot directly use the definitions $a_{\alpha}^{(k)+}=\max _{C_{\alpha}}\left\{\frac{\partial H}{\partial x^{(k)}}\left(\nabla \varphi_{\alpha}\right), 0\right\}$ etc., 
where the maximum is taken over the spatial domain $C_{\alpha}$ (see (3.2)). We must translate this definition into a maximum over the range of function values. For example, in two dimensions, we define

$$
\begin{aligned}
& a^{+}=\max _{\substack{u \in I\left(u^{-}, u^{+}\right) \\
\mathcal{C} \leq v \leq \mathcal{D}^{+}}}\left\{H_{x}(u, v), 0\right\}, a^{-}=\left|\min _{\substack{u \in I\left(u^{-}, u^{+}\right) \\
\mathcal{C} \leq v \leq \mathcal{D}}}\left\{H_{x}(u, v), 0\right\}\right| \\
& b^{+}=\max _{\substack{\mathcal{A} \leq u \leq \mathcal{B} \\
v \in J\left(v^{-}, v^{+}\right)}}\left\{H_{y}(u, v), 0\right\}, b^{-}=\left|\min _{\substack{\mathcal{A} \leq u \leq \mathcal{B} \\
v \in I\left(v^{-}, v^{+}\right)}}\left\{H_{y}(u, v), 0\right\}\right|,
\end{aligned}
$$

where $[\mathcal{A}, \mathcal{B}]$ is the range of $u$ and $[\mathcal{C}, \mathcal{D}]$ is the range of $v$. With such a choice of $a$ and $b$ (and similarly in more than two space dimensions) the multi-dimensional flux approximation is monotone.

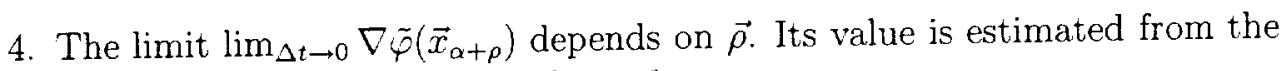
reconstruction that corresponds to $\vec{\rho}$.

5. When $a_{\alpha}^{(k)+}$ and $a_{\alpha}^{(k)-}$ are replaced by $a_{\alpha}^{(k)}=\max _{C_{\alpha}}\left\{\left|\frac{\partial H}{\partial x^{(k)}}\left(\nabla \varphi_{\alpha}\right)\right|\right\}$, $V_{\alpha}=2^{d} \prod_{k=1}^{d} a_{\alpha}^{(k)}=2^{d}\left|\vec{v}_{\alpha}^{\bar{\rho}}\right|$. In this case the semi-discrete scheme (3.6) becomes

$$
\frac{d}{d t} \varphi_{\alpha}(t)=-\frac{1}{2^{d}} \sum_{\rho} H\left(\nabla \tilde{\varphi}_{\alpha+\rho}^{n}\right)+\frac{1}{2} \sum_{k=1}^{d} a_{\alpha}^{(k)}\left(\varphi_{x^{(k)}}^{+}-\varphi_{x^{(k)}}^{-}\right)
$$

A simpler one- and two-dimensional version of (3.7) was presented in [20] with a less accurate estimate of the local speed of propagation, $a=\max _{k} a^{(k)}$.

6. In practice, the speeds of propagation is estimated from the reconstruction of $\nabla \tilde{\varphi}_{\alpha+\rho}^{n}$, i.e.,

$$
a_{\alpha}^{(k)+}=\max _{\rho}\left\{\frac{\partial H}{\partial x^{(k)}}\left(\nabla \tilde{\varphi}_{\alpha+\rho}\right), 0\right\}, \quad a_{\alpha}^{(k)-}=\left|\min _{\rho}\left\{\frac{\partial H}{\partial x^{(k)}}\left(\nabla \tilde{\varphi}_{\alpha+\rho}\right), 0\right\}\right| .
$$

\subsection{Two and Three Dimensional Schemes}

For convenience, we write out (3.6) in two and three dimensions. In two dimensions, we let $\alpha=(i, j)$ with coordinate notation $\left(x_{i}, y_{j}\right)$, and let the local speeds of propagation be $\left(a_{i, j}^{ \pm}, b_{i, j}^{ \pm}\right):=\left(a_{\alpha}^{(1) \pm}, a_{\alpha}^{(2) \pm}\right)$. Explicitly, for convex $H$ we use the estimates

$$
\begin{array}{ll}
a_{i, j}^{+}=\max _{ \pm}\left\{H_{x}\left(\varphi_{x}^{ \pm}, \varphi_{y}^{ \pm}\right), 0\right\}, \quad a_{i, j}^{-}=\left|\min _{ \pm}\left\{H_{x}\left(\varphi_{x}^{ \pm}, \varphi_{y}^{ \pm}\right), 0\right\}\right|, \\
b_{i, j}^{+}=\max _{ \pm}\left\{H_{y}\left(\varphi_{x}^{ \pm}, \varphi_{y}^{ \pm}\right), 0\right\}, \quad b_{i, j}^{-}=\left|\min _{ \pm}\left\{H_{y}\left(\varphi_{x}^{ \pm}, \varphi_{y}^{ \pm}\right), 0\right\}\right|,
\end{array}
$$


where the max and min are taken over all permutations of \pm . Then (3.6) becomes (suppressing the indices $i, j$ )

$$
\begin{aligned}
\frac{d \varphi}{d t} & =\frac{a^{+} a^{-}}{\left(a^{+}+a^{-}\right)}\left(\varphi_{x}^{+}-\varphi_{x}^{-}\right)+\frac{b^{+} b^{-}}{\left(b^{+}+b^{-}\right)}\left(\varphi_{y}^{+}-\varphi_{y}^{-}\right) \\
& -\frac{a^{-} b^{-} H\left(\varphi_{x}^{+}, \varphi_{y}^{+}\right)+a^{+} b^{-} H\left(\varphi_{x}^{-}, \varphi_{y}^{+}\right)+a^{-} b^{+} H\left(\varphi_{x}^{+}, \varphi_{y}^{-}\right)+a^{+} b^{+} H\left(\varphi_{x}^{-}, \varphi_{y}^{-}\right)}{\left(a^{+}+a^{-}\right)\left(b^{+}+b^{-}\right)}
\end{aligned}
$$

If we replace $a_{i, j}^{+}$and $a_{i, j}^{-}$by $a_{i, j}=\max _{ \pm}\left|\left\{H_{x}\left(\varphi_{x}^{ \pm}, \varphi_{y}^{ \pm}\right)\right\}\right|$and similarly $b_{i, j}^{+}$and $b_{i, j}^{-}$ by $b_{i, j}=\max _{ \pm}\left|\left\{H_{y}\left(\varphi_{x}^{ \pm}, \varphi_{y}^{ \pm}\right)\right\}\right|$, then (3.9) can be further simplified to (compare with $[20$, Eq. (5.10)])

$$
\begin{aligned}
\frac{d \varphi_{i, j}}{d t} & =\frac{a_{i, j}}{2}\left(\varphi_{x}^{+}-\varphi_{x}^{-}\right)+\frac{b_{i, j}}{2}\left(\varphi_{y}^{+}-\varphi_{y}^{-}\right) \\
& -\frac{1}{4}\left[H\left(\varphi_{x}^{+}, \varphi_{y}^{+}\right)+H\left(\varphi_{x}^{-}, \varphi_{y}^{+}\right)+H\left(\varphi_{x}^{+}, \varphi_{y}^{-}\right)+H\left(\varphi_{x}^{-}, \varphi_{y}^{-}\right)\right] .
\end{aligned}
$$

In three dimensions, we let $\alpha=(i, j, k)$ with coordinate notation $\left(x_{i}, y_{j}, z_{k}\right)$, and let the local speeds of propagation be $\left(a_{i, j, k}^{ \pm}, b_{i, j, k}^{ \pm}, c_{i, j, k}^{ \pm}\right):=\left(a_{\alpha}^{(1) \pm}, a_{\alpha}^{(2) \pm}, a_{\alpha}^{(3) \pm}\right)$. Thus $a$ and $b$ are the obvious generalization of (3.8), and $c$ is estimated as

$$
c_{i, j, k}^{+}=\max _{ \pm}\left\{H_{z}\left(\varphi_{x}^{ \pm}, \varphi_{y}^{ \pm}, \varphi_{z}^{ \pm}\right), 0\right\}, \quad c_{i, j, k}^{-}=\left|\min _{ \pm}\left\{H_{z}\left(\varphi_{x}^{ \pm}, \varphi_{y}^{ \pm}, \varphi_{z}^{ \pm}\right), 0\right\}\right| .
$$

Then the semi-discrete scheme becomes (suppressing the indices $i, j, k$ )

$$
\begin{aligned}
\frac{d \varphi}{d t} & =-\frac{1}{\left(a^{+}+a^{-}\right)\left(b^{+}+b^{-}\right)\left(c^{+}+c^{-}\right)} \\
& \cdot\left[a^{-} b^{-} c^{-} H\left(\varphi_{x}^{+}, \varphi_{y}^{+}, \varphi_{z}^{+}\right)+a^{-} b^{-} c^{+} H\left(\varphi_{x}^{+}, \varphi_{y}^{+}, \varphi_{z}^{-}\right)+a^{-} b^{+} c^{-} H\left(\varphi_{x}^{+}, \varphi_{y}^{-}, \varphi_{z}^{+}\right)\right. \\
& +a^{-} b^{+} c^{+} H\left(\varphi_{x}^{+}, \varphi_{y}^{-}, \varphi_{z}^{-}\right)+a^{+} b^{-} c^{-} H\left(\varphi_{x}^{-}, \varphi_{y}^{+}, \varphi_{z}^{+}\right)+a^{+} b^{-} c^{+} H\left(\varphi_{x}^{-}, \varphi_{y}^{+}, \varphi_{z}^{-}\right) \\
& \left.+a^{+} b^{+} c^{-} H\left(\varphi_{x}^{-}, \varphi_{y}^{-}, \varphi_{z}^{+}\right)+a^{+} b^{+} c^{+} H\left(\varphi_{x}^{-}, \varphi_{y}^{-}, \varphi_{z}^{-}\right)\right] \\
& +\frac{a^{+} a^{-}}{\left(a^{+}+a^{-}\right)}\left(\varphi_{x}^{+}-\varphi_{x}^{-}\right)+\frac{b^{+} b^{-}}{\left(b^{+}+b^{-}\right)}\left(\varphi_{y}^{+}-\varphi_{y}^{-}\right)+\frac{c^{+} c^{-}}{\left(c^{+}+c^{-}\right)}\left(\varphi_{z}^{+}-\varphi_{z}^{-}\right) .
\end{aligned}
$$

The three-dimensional scheme (3.11) can be further simplified by replacing $a_{i, j}^{+}, a_{i, j}^{-}$by $a_{i, j}$ and $b_{i, j}^{+}, b_{i, j}^{-}$by $b_{i, j}$ similarly to the two-dimensional case, and also replacing $c_{i, j}^{+}, c_{i, j}^{-}$ by $c_{i, j}=\max _{ \pm}\left|\left\{H_{z}\left(\varphi_{x}^{ \pm}, \varphi_{y}^{ \pm}, \varphi_{z}^{ \pm}\right)\right\}\right|$. In this case

$$
\begin{aligned}
\frac{d \varphi_{i, j}}{d t} & =\frac{a_{i, j}}{2}\left(\varphi_{x}^{+}-\varphi_{x}^{-}\right)+\frac{b_{i, j}}{2}\left(\varphi_{y}^{+}-\varphi_{y}^{-}\right)+\frac{c_{i, j}}{2}\left(\varphi_{z}^{+}-\varphi_{z}^{-}\right) \\
& -\frac{1}{8}\left[H\left(\varphi_{x}^{+}, \varphi_{y}^{+}, \varphi_{z}^{+}\right)+H\left(\varphi_{x}^{+}, \varphi_{y}^{+}, \varphi_{z}^{-}\right)+H\left(\varphi_{x}^{+}, \varphi_{y}^{-}, \varphi_{z}^{+}\right)+H\left(\varphi_{x}^{+}, \varphi_{y}^{-}, \varphi_{z}^{-}\right)\right. \\
& \left.+H\left(\varphi_{x}^{-}, \varphi_{y}^{+}, \varphi_{z}^{+}\right)+H\left(\varphi_{x}^{-}, \varphi_{y}^{+}, \varphi_{z}^{-}\right)+H\left(\varphi_{x}^{-}, \varphi_{y}^{-}, \varphi_{z}^{+}\right)+H\left(\varphi_{x}^{-}, \varphi_{y}^{-}, \varphi_{z}^{-}\right)\right]
\end{aligned}
$$




\subsection{A Dimension-by-Dimension 5th-order Reconstruction}

The reconstructions $\nabla \tilde{\varphi}_{\alpha+\rho}^{n}$ can be easily computed in a direction-by-direction manner. Such a direction-by-direction reconstruction is commonly used in upwind schemes [15], and we have used this strategy with central schemes in [6]. Here we show a threedimensional example; generalizing this technique to more dimensions is straightforward. Using the notation of Section 2.2, a three-dimensional fifth-order reconstruction is

- For each $j, k: \varphi_{x}^{ \pm}=$reconstruct_$_{-} \varphi^{\prime}\left( \pm, \varphi_{* j, k}\right)$

- For each $i, k: \varphi_{y}^{ \pm}=$reconstruct $-\varphi^{\prime}\left( \pm, \varphi_{i, *, k}\right)$

- For each $i, j: \varphi_{z}^{ \pm}=$reconstruct_- $\varphi^{\prime}\left( \pm, \varphi_{i, j, *}\right)$

where the subscript ' $*$ ' denotes the full range of an index: $\varphi_{*, j, k}$ denotes the array $\left(\varphi_{1, j, k}, \ldots, \varphi_{N, j, k}\right)$, etc. We denote this operation in three dimensions as

$$
\nabla \tilde{\varphi}^{ \pm}=\left(\varphi_{x}^{ \pm}, \varphi_{x}^{ \pm}, \varphi_{x}^{ \pm}\right)=\text {reconstruct }-\nabla \varphi( \pm, \varphi) .
$$

The results of this operation are three-dimensional arrays with elements $\left(\varphi_{x}^{ \pm}\right)_{i, j, k}$, $\left(\varphi_{y}^{ \pm}\right)_{i, j, k}$ and $\left(\varphi_{z}^{ \pm}\right)_{i, j, k}$.

Using this notation, we can turn Algorithm 2.1 into a three-dimensional scheme: replace reconstruct_ $\varphi^{\prime}\left( \pm, \varphi^{n}\right)$ with reconstruct_$\nabla \varphi( \pm, \varphi)$, and let $F$ denotes the right hand side of (3.11). Applying this modified version of Algorithm 2.1 to each grid node gives a three-dimensional scheme that is fifth-order in space and fourth-order in time.

\section{Numerical Simulations}

In this section we present simulations that demonstrate the features of the schemes we developed in the previous sections. The scheme we test is the fifth-order semi-discrete method in one (Section 4.1), two (Section 4.2), and three (Section 4.4) space dimensions. Some of these examples are standard test cases that can be found, e.g., in $[20,28,32]$. In Section 4.3 we present a numerical stability study in two space dimensions.

We do not follow the practice in [15] of masking singular regions from our error measurements, as we prefer to include the entire domain in our error estimate.

\subsection{One-Dimensional Examples}

\section{A convex Hamiltonian}

We start by testing the performance of our schemes in a convex problem. We approximate solutions of the one-dimensional equation

$$
\phi_{t}+\frac{1}{2}\left(\phi_{x}+1\right)^{2}=0
$$

subject to the initial data $\phi(x, 0)=-\cos (\pi x)$ with periodic boundary conditions on $[0,2]$. The change of variables, $u(x, t)=\phi_{x}(x, t)+1$, transforms the equation into 
the Burgers' equation, $u_{t}+\frac{1}{2}\left(u^{2}\right)_{x}=0$, which can be easily solved via the method of characteristics [32]. The solution develops a singularity in the form of a discontinuous derivative at time $t=\pi^{-2}$.

The results of our simulations are shown in Figure 4.1. The order of accuracy of these methods is determined from the relative $L^{1}$ error, defined as the $L^{1}$-norm of the error divided by the $L^{1}$-norm of the exact solution. These results along with the relative $L^{\infty}$-norm before the singularity at $T=0.8 / \pi^{2}$, and after the singularity at $T=1.5 / \pi^{2}$ are given in Table 4.1 .

\begin{tabular}{|c||c|c|c|c||}
\hline \multirow{2}{*}{$N$} & \multicolumn{5}{c||}{ Before singularity $T=0.8 / \pi^{2}$} \\
\cline { 2 - 5 } & relative $L^{1}$-error & $L^{1}$-order & relative $L^{\infty}$-error & $L^{\infty}$-order \\
\hline \hline 100 & $2.78 \times 10^{-6}$ & - & $5.74 \times 10^{-7}$ & - \\
\hline 200 & $9.89 \times 10^{-8}$ & 4.81 & $1.14 \times 10^{-8}$ & 5.65 \\
\hline 400 & $3.20 \times 10^{-9}$ & 4.95 & $1.92 \times 10^{-10}$ & 5.90 \\
\hline 800 & $1.01 \times 10^{-10}$ & 4.99 & $3.04 \times 10^{-12}$ & 5.98 \\
\hline \hline & \multicolumn{5}{|c||}{ After singularity T $=1.5 / \pi^{2}$} \\
\cline { 2 - 5 }$N$ & relative $L^{1}$-error & $L^{1}$-order & relative $L^{\infty}$-error & $L^{\infty}$-order \\
\hline \hline 100 & $2.04 \times 10^{-4}$ & - & $2.02 \times 10^{-4}$ & - \\
\hline 200 & $7.21 \times 10^{-7}$ & 9.60 & $8.15 \times 10^{-7}$ & 9.60 \\
\hline 400 & $2.64 \times 10^{-5}$ & -5.19 & $-5.19 \times 10^{-5}$ & -6.65 \\
\hline 800 & $2.55 \times 10^{-5}$ & 0.05 & $0.05 \times 10^{-5}$ & 0.10 \\
\hline
\end{tabular}

Table 4.1: Relative $L^{1}$-errors for the one-dimensional convex HJ problem (4.1) before $\left(T=0.8 / \pi^{2}\right)$ and after $\left(T=1.5 / \pi^{2}\right)$ the singularity formation.

\section{A non-convex Hamiltonian}

In this example we deal with non-convex Hamilton-Jacobi equations. In one dimension we solve

$$
\phi_{t}-\cos \left(\phi_{x}+1\right)=0
$$

subject to the initial data $\phi(x, 0)=-\cos (\pi x)$ with periodic boundary conditions on $[0,2]$. In this case $(4.2)$ has a smooth solution for $t \lesssim 1.049 / \pi^{2}$, after which a singularity forms. A second singularity forms at $t \approx 1.29 / \pi^{2}$. The results are shown in Figures 4.2 . The convergence results before and after the singularity formation are given in Table 4.2 .

Remark. Tables 4.1 and 4.2 show that after the singularity formation the order of convergence deteriorates. In the following examples we will see that while a close examination of the convergence properties confirms this observation, in all the cases we examined, the error of the fifth-order semi-discrete scheme is less than the error of the other two published fifth-order methods for $\mathrm{HJ}$ equations $[6,15]$. 

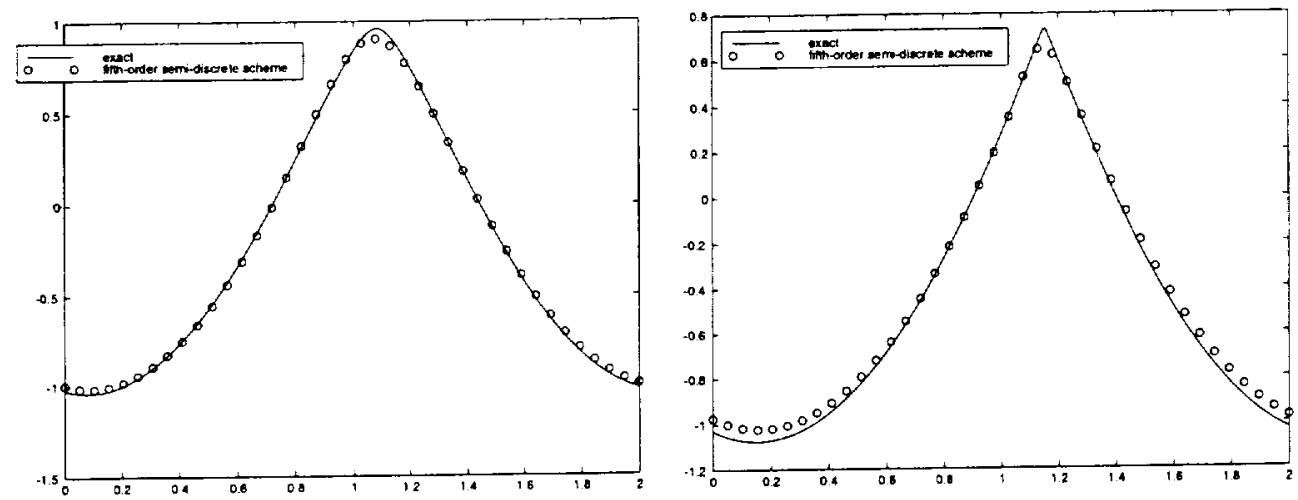

Figure 4.1: One-dimensional convex Hamiltonian (4.1). Left: the solution before the singularity formation, $T=0.8 / \pi^{2}$. Right: the solution after the singularity formation, $T=1.5 / \pi^{2} . \quad N=40$. The fifth-order approximation is plotted on top of the exact solution.

\begin{tabular}{|c||c|c|c|c||}
\hline \multicolumn{1}{|c||}{$N$} & \multicolumn{5}{c||}{ Before singularity $T=0.8 / \pi^{2}$} \\
\cline { 2 - 5 } & relative $L^{1}$-error & $L^{1}$-order & relative $L^{\infty}$-error & $L^{\infty}$-order \\
\hline \hline 100 & $1.20 \times 10^{-6}$ & - & $4.24 \times 10^{-7}$ & - \\
\hline 200 & $5.29 \times 10^{-8}$ & 4.50 & $2.18 \times 10^{-8}$ & 4.28 \\
\hline 400 & $2.14 \times 10^{-9}$ & 4.62 & $6.06 \times 10^{-10}$ & 5.17 \\
\hline 800 & $8.24 \times 10^{-11}$ & 4.70 & $1.17 \times 10^{-11}$ & 5.69 \\
\hline \hline \multirow{3}{*}{$N$} & \multicolumn{4}{|c||}{ After singularity T $=1.5 / \pi^{2}$} \\
\cline { 2 - 5 } & relative $L^{1}$-error & $L^{1}$-order & relative $L^{\infty}$-error & $L^{\infty}$-order \\
\hline \hline 100 & $1.91 \times 10^{-5}$ & - & $3.52 \times 10^{-5}$ & - \\
\hline 200 & $4.98 \times 10^{-5}$ & -1.38 & $4.27 \times 10^{-5}$ & -0.27 \\
\hline 400 & $2.91 \times 10^{-6}$ & 4.10 & $2.47 \times 10^{-6}$ & 4.11 \\
\hline 800 & $4.20 \times 10^{-6}$ & -0.53 & $3.63 \times 10^{-6}$ & -0.56 \\
\hline
\end{tabular}

Table 4.2: Relative $L^{1}$-errors for the one-dimensional non-convex HJ problem (4.2) before $\left(T=0.8 / \pi^{2}\right)$ and after $\left(T=1.5 / \pi^{2}\right)$ the singularity formation. 

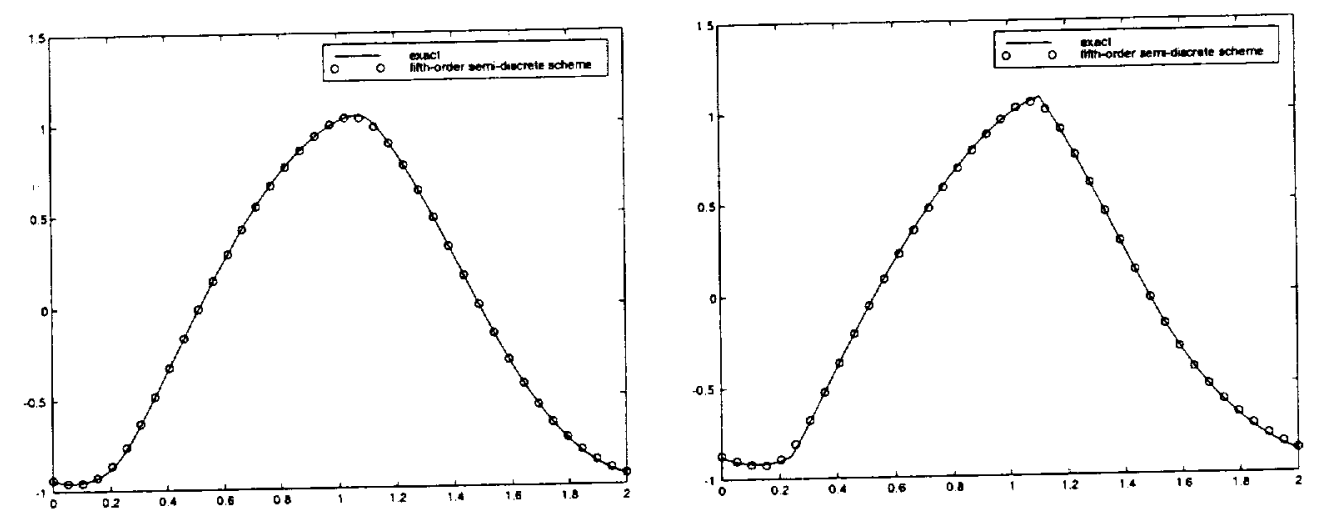

Figure 4.2: One-dimensional non-convex Hamiltonian (4.2). Left: The solution before the singularity formation, $T=0.8 / \pi^{2}$. Right: The solution after the singularity formation, $T=1.5 / \pi^{2} . N=40$. The fifth-order approximation is plotted on top of the exact solution.

\subsubsection{A comparison with existing fifth-order WENO-based methods}

In Figure 4.3 we compare the error of our new fifth-order semi-discrete scheme (Algorithm 2.1) with our fully-discrete scheme [6], and with the upwind WENO method of [15] (with a local Lax-Friedrichs flux). We also present results obtained with the method of [15] where the local Lax-Friedrichs flux was replaced by the semi-discrete central flux (2.7), which compares our smoothness measures with those of [15].

We see that before the singularity formation the $L^{1}$-error of our semi-discrete method is as much as an order of magnitude smaller than the $L^{1}$-error of the methods in [6] and [15]. The method of [15] with the flux (2.7) yields somewhat smaller errors for large grid spacing for the convex Hamiltonian, but becomes comparable to our method as the grid spacing decreases. For the non-convex Hamiltonian the method of [15] with flux (2.7) has larger errors than Algorithm 2.1. We take this as indication that the smoothness measures in [15] may be slightly better for large grid spacing and some Hamiltonians.

After the formation of the singularity the behavior of the error in both methods that are based on the flux (2.7) is more erratic than the other two methods. Nonetheless, the methods that use the flux (2.7) have errors that are sometimes dramatically smaller than the other two methods. At no time is the error of methods using the flux $(2.7)$ larger than that of the other two methods. Further comparisons are done in the next example and in Section 4.3. A theoretical study of the convergence of these schemes is beyond the scope of this work and is left for the future.

\section{A linear advection equation}

In this example ([15] with a misprint, corrected in [34]) we solve the one-dimensional linear advection equation, i.e., $H\left(\phi_{x}\right)=\phi_{x}$. We assume periodic boundary conditions 

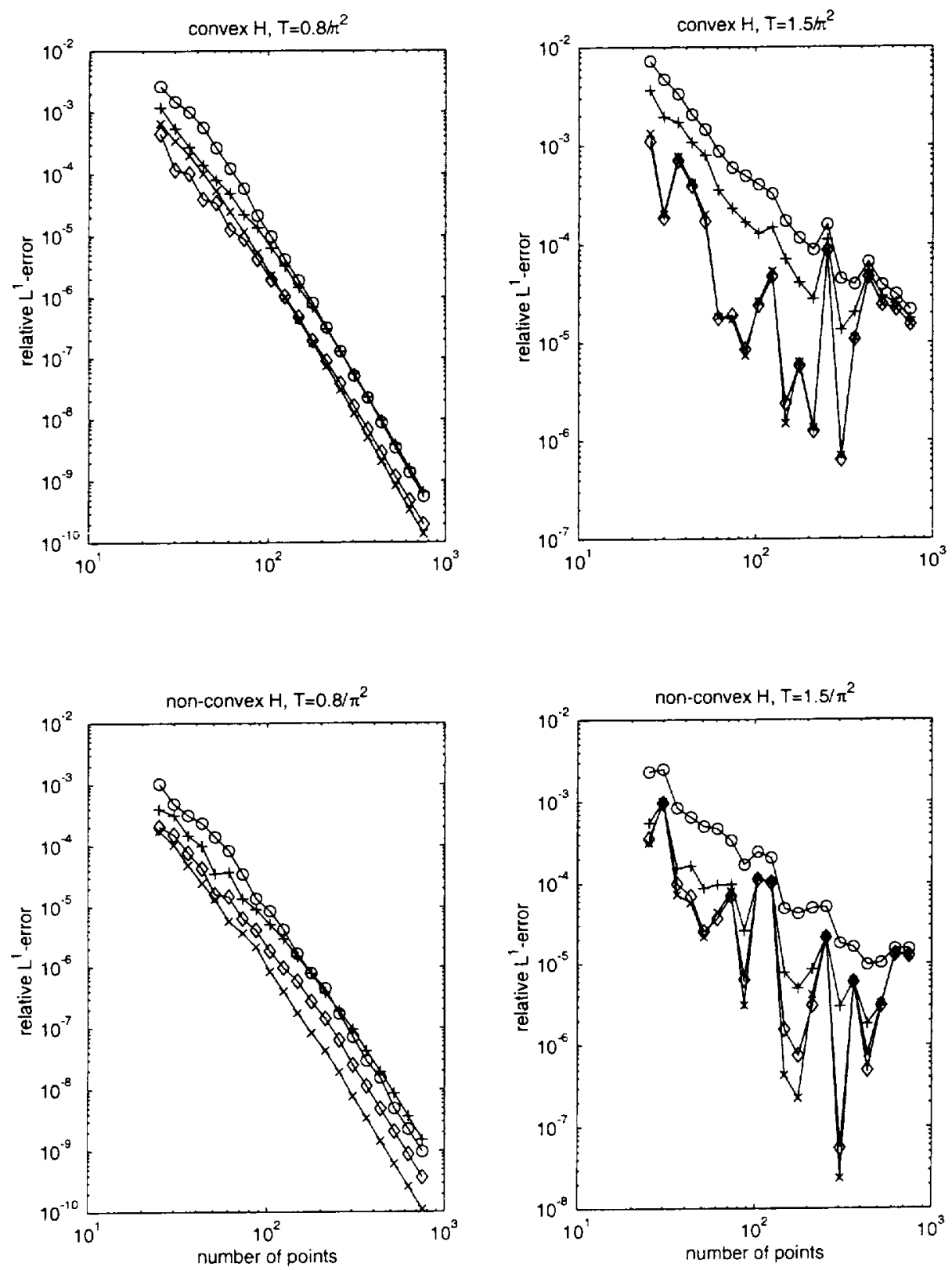

Figure 4.3: Convergence results for the convex Hamiltonian (4.1) (top) and non-convex Hamiltonian (4.2) (bottom). The relative $L^{1}$-error are plotted against the number of grid nodes. "x": our semi-discrete fifth-order method (Algorithm 2.1). "+": the fifth-order method of [6]. "o": the fifth-order method of [15] with a local Lax-Friedrichs flux. " $\diamond$ ": the fifth-order method of $[15]$ with the flux (2.7). Left: Before the singularity. Right: After the singularity. 
on $[-1,1]$, and take the initial data as $\phi(x, 0)=g(x-0.5)$ on $[-1,1]$, where

$$
\begin{aligned}
& g(x)=-\left(\frac{\sqrt{3}}{2}+\frac{9}{2}+\frac{2 \pi}{3}\right)(x+1)+h(x), \\
& h(x)= \begin{cases}2 \cos \left(\frac{3 \pi}{2} x^{2}\right)-\sqrt{3}, & -1<x<-\frac{1}{3}, \\
3 / 2+3 \cos (2 \pi x), & -\frac{1}{3}<x<0, \\
15 / 2-3 \cos (2 \pi x), & 0<x<\frac{1}{3}, \\
(28+4 \pi+\cos (3 \pi x)) / 3+6 \pi x(x-1), & \frac{1}{3}<x<1 .\end{cases}
\end{aligned}
$$

The results of our semi-discrete fifth-order method (Algorithm 2.1) are shown in Figure 4.4, where it is compared with the fifth-order methods of [6] and [15]. The semidiscrete method (Algorithm 2.1) shows reduced dissipation compared to the method in [15]. In [6] we showed that the fully-discrete fifth-order method we developed there is more stable than the method of [15] from the point of view of being able to use larger time steps. The numerical results here are based on fitting to each scheme its optimal time-step, hence the reduced dissipation for the fully-discrete scheme [6].

\subsection{Two-Dimensional Examples}

\section{A convex Hamiltonian}

In two dimensions we solve a problem similar to (4.1)

$$
\phi_{t}+\frac{1}{2}\left(\phi_{x}+\phi_{y}+1\right)^{2}=0
$$

which can be reduced to a one-dimensional problem via the coordinate transformation $\left(\begin{array}{l}\xi \\ \eta\end{array}\right)=\left(\begin{array}{cc}1 / 2 & 1 / 2 \\ 1 / 2 & -1 / 2\end{array}\right)\left(\begin{array}{l}x \\ y\end{array}\right)$. The results of the second-order calculations for the initial data $\phi(x, y, 0)=-\cos (\pi(x+y) / 2)=-\cos (\pi \xi)$ are shown in Figure 4.5 . The convergence rates for the two-dimensional fifth-order scheme (3.9) before and after the singularity are shown in Table 4.3 .

\section{A non-convex Hamiltonian}

The two-dimensional non-convex problem, which is analogous to the one-dimensional problem (4.2), is

$$
\phi_{t}-\cos \left(\phi_{x}+\phi_{y}+1\right)=0 \text {. }
$$

We assume the initial data $\phi(x, y, 0)=-\cos (\pi(x+y) / 2)$, and periodic boundary conditions. The results are shown in Figure 4.6. The convergence results for the twodimensional fifth-order scheme (3.9) before and after the singularity formation are given in Table 4.4 . 

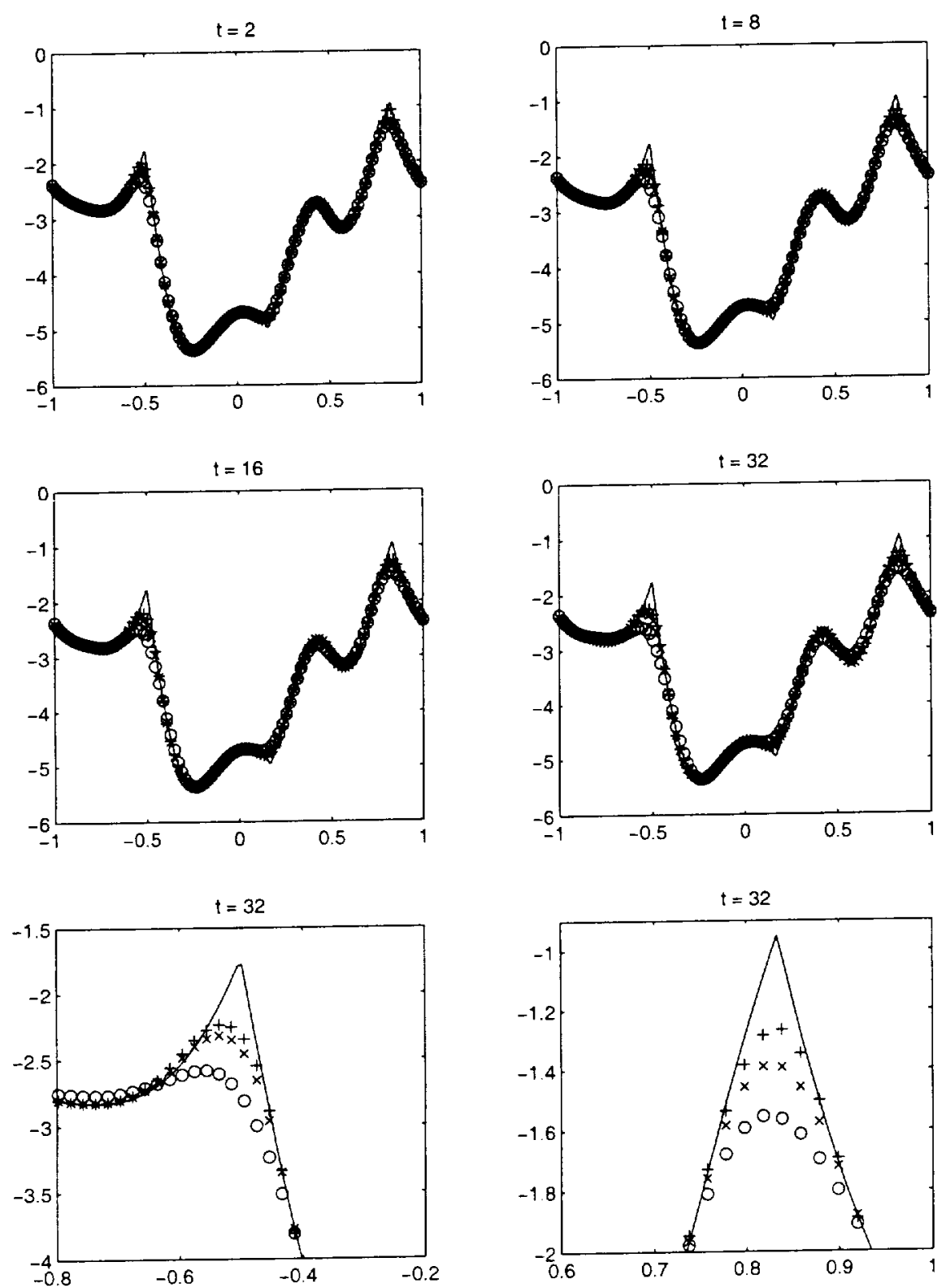

Figure 4.4: One-dimensional linear advection, (4.3). $T=2,8,16,32 . \quad N=100$. " $\mathrm{x}$ ": our semi-discrete fifth-order method (Algorithm 2.1). "+": the fifth-order method of [6]. "o": the fifth-order method of [15] with a local Lax-Friedrichs flux. In the bottom two pictures we zoom on two of the peaks in the solution at $T=32$. 


\begin{tabular}{|c||c|c||c|c||}
\hline \multicolumn{5}{|c|}{ Before singularity $T=0.8 / \pi^{2}$} \\
\hline$N$ & relative $L^{1}$-error & $L^{1}$-order & relative $L^{\infty}$-error & $L^{\infty}$-order \\
\hline \hline 50 & $3.38 \times 10^{-5}$ & - & $3.66 \times 10^{-7}$ & - \\
\hline 100 & $1.90 \times 10^{-6}$ & 4.15 & $5.30 \times 10^{-9}$ & 6.11 \\
\hline 200 & $7.35 \times 10^{-8}$ & 4.69 & $6.02 \times 10^{-11}$ & 6.46 \\
\hline \multicolumn{5}{|c|}{ After singularity $T=1.5 / \pi^{2}$} \\
\hline$N$ & relative $L^{1}$-error & $L^{1}$-order & relative $L^{\infty}$-error & $L^{\infty}$-order \\
\hline \hline 50 & $8.68 \times 10^{-4}$ & - & $1.60 \times 10^{-5}$ & - \\
\hline 100 & $3.06 \times 10^{-4}$ & 1.50 & $2.88 \times 10^{-6}$ & 2.47 \\
\hline 200 & $2.77 \times 10^{-5}$ & 3.46 & $8.29 \times 10^{-8}$ & 5.12 \\
\hline
\end{tabular}

Table 4.3: Relative $L^{1}$ - and $L^{\infty}$-errors for the two-dimensional convex HJ problem (4.4) before and after singularity formation, computed with (3.9) integrated in time as in Algorithm 2.1, with the fifth-order reconstruction of Section 3.3 .
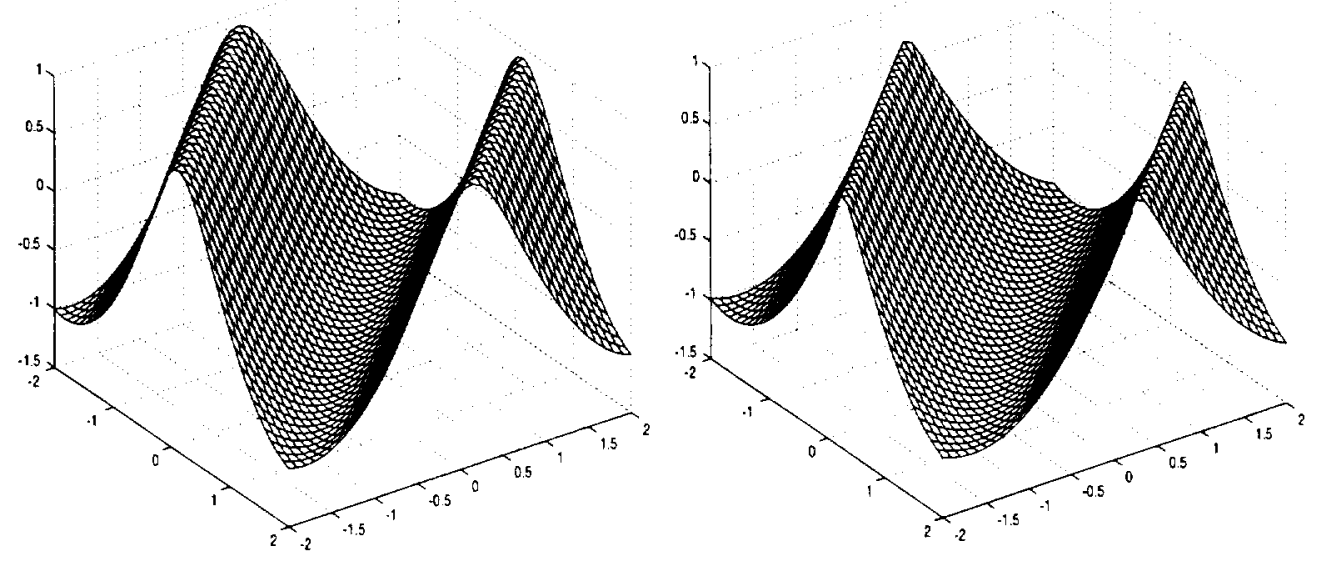

Figure 4.5: Two-dimensional convex Hamiltonian, (4.4). Left: the solution before the singularity formation, $T=0.8 / \pi^{2}$. Right: the solution after the singularity formation, $T=1.5 / \pi^{2} . N=40 \times 40$. The solution is computed with (3.9) integrated in time as in Algorithm 2.1, with the fifth-order reconstruction of Section 3.3 . 


\begin{tabular}{|c||c|c||c|c||}
\hline \multicolumn{5}{|c|}{ Before singularity $T=0.8 / \pi^{2}$} \\
\hline$N$ & relative $L^{1}$-error & $L^{1}$-order & relative $L^{\infty}$-error & $L^{\infty}$-order \\
\hline \hline 50 & $1.70 \times 10^{-5}$ & - & $6.04 \times 10^{-8}$ & - \\
\hline 100 & $1.69 \times 10^{-6}$ & 3.33 & $5.20 \times 10^{-9}$ & 3.54 \\
\hline 200 & $8.16 \times 10^{-8}$ & 4.37 & $1.17 \times 10^{-10}$ & 5.47 \\
\hline \multicolumn{5}{|c|}{ After singularity $T=1.5 / \pi^{2}$} \\
\hline$N$ & relative $L^{1}$-error & $L^{1}$-order & relative $L^{\infty}$-error & $L^{\infty}$-order \\
\hline \hline 50 & $2.63 \times 10^{-3}$ & - & $9.55 \times 10^{-6}$ & - \\
\hline 100 & $3.40 \times 10^{-4}$ & 2.95 & $1.52 \times 10^{-6}$ & 2.65 \\
\hline 200 & $7.20 \times 10^{-5}$ & 2.24 & $2.72 \times 10^{-7}$ & 2.49 \\
\hline
\end{tabular}

Table 4.4: Relative $L^{1}$ - and $L^{\infty}$-errors for the two-dimensional non-convex HJ problem (4.5) before and after the singularity formation, computed with (3.9) integrated in time as in Algorithm 2.1, with the fifth-order reconstruction of Section 3.3.
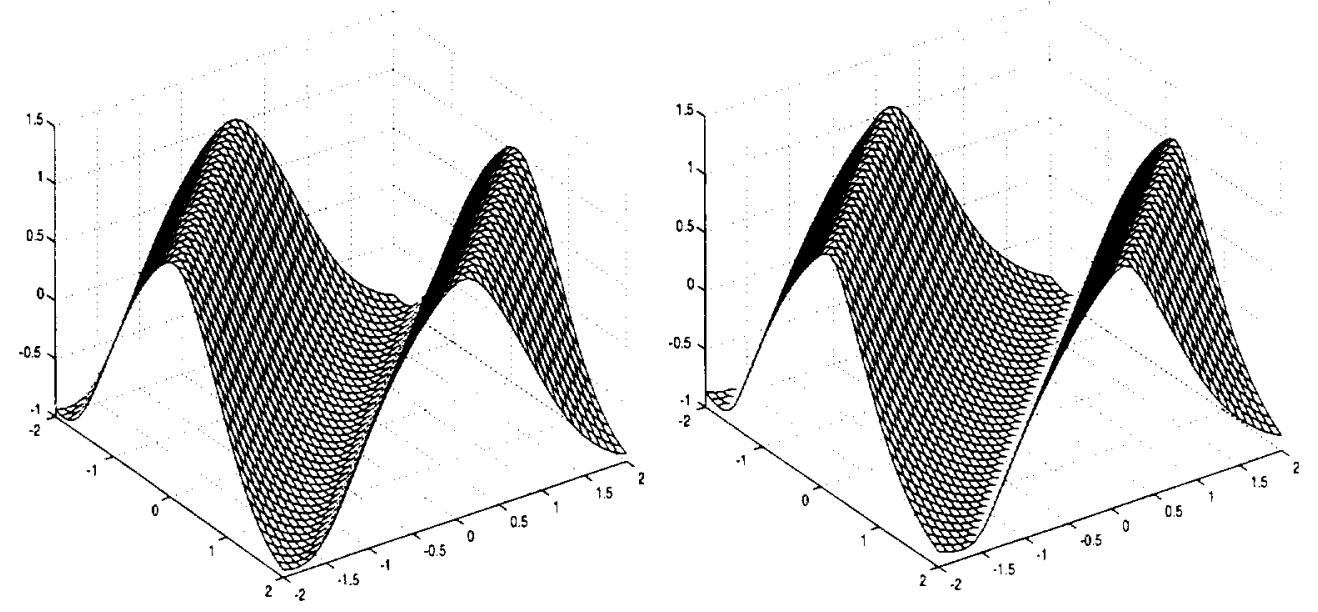

Figure 4.6: Two-dimensional non-convex Hamiltonian, (4.4). Left: the solution before the singularity formation, $T=0.8 / \pi^{2}$. Right: the solution after the singularity formation, $T=1.5 / \pi^{2} . N=40 \times 40$. The solution is computed with (3.9) integrated in time as in Algorithm 2.1, with the fifth-order reconstruction of Section 3.3. 


\section{A fully two-dimensional example}

To check the performance of our method on fully two-dimensional problems we solve a test problem which we introduced in [4]:

$$
\phi_{t}+\phi_{x} \phi_{y}=0, \quad(x, y) \in[-\pi, \pi] \times[-\pi, \pi]
$$

subject to the initial data $\phi(x, y, 0)=\sin (x)+\cos (y)$ and to periodic boundary conditions. The exact solution for this problem is given implicitly by $\phi(x, y, t)=$ $-\cos (q) \sin (r)+\sin (q)+\cos (r)$ where $x=q-t \sin (r)$ and $y=r+t \cos (q)$. This solution is smooth for $t<1$, continuous for all $t$ and has discontinuous derivatives for $t \geq 1$. The results of our simulations at times $T=0.8,1.5$, are shown in Figure 4.7. For comparison we show in Figure 4.8 the results obtained for the same problem with our fully-discrete method [6]. The convergence results for the fifth-order method (3.9) before the singularity formation are given in Table 4.5 and confirm the expected order of accuracy.

\begin{tabular}{|c||c|c||c|c||}
\hline \multicolumn{5}{|c|}{ Before singularity $T=0.8$} \\
\hline$N$ & relative $L^{1}$-error & $L^{1}$-order & relative $L^{\infty}$-error & $L^{\infty}$-order \\
\hline \hline 50 & $2.39 \times 10^{-6}$ & - & $1.34 \times 10^{-8}$ & - \\
\hline 100 & $8.52 \times 10^{-8}$ & 4.81 & $1.40 \times 10^{-10}$ & 6.57 \\
\hline 200 & $3.05 \times 10^{-9}$ & 4.80 & $1.24 \times 10^{-12}$ & 6.83 \\
\hline
\end{tabular}

Table 4.5: Relative $L^{1}$-errors for the two-dimensional HJ problem (4.6) before the singularity formation. $T=0.8$. The solution is computed with (3.9) integrated in time as in Algorithm 2.1, with the fifth-order reconstruction of Section 3.3.

\section{An eikonal equation in geometric optics}

We consider a two-dimensional non-convex problem that arises in geometric optics [17]

$$
\left\{\begin{array}{l}
\phi_{t}+\sqrt{\phi_{x}^{2}+\phi_{y}^{2}+1}=0 \\
\phi(x, y, 0)=\frac{1}{4}(\cos (2 \pi x)-1)(\cos (2 \pi y)-1)-1
\end{array}\right.
$$

The results of our fifth-order method at time $T=0.6$ are shown in Figure 4.9, where we see the sharp corners that develop in this problem.

\section{An optimal control problem}

We solve an optimal control problem related to cost determination [32]. Here the Hamiltonian is of the form $H(x, y, \nabla \phi)$ :

$$
\left\{\begin{array}{l}
\phi_{t}-\sin (y) \phi_{x}+\sin (x) \phi_{y}+\left|\phi_{y}\right|-\frac{1}{2} \sin ^{2}(y)-1+\cos (x)=0 \\
\phi(x, y, 0)=0
\end{array}\right.
$$

The result of our fifth-order semi-discrete scheme at time $T=1$ is shown in Figure 4.10. 


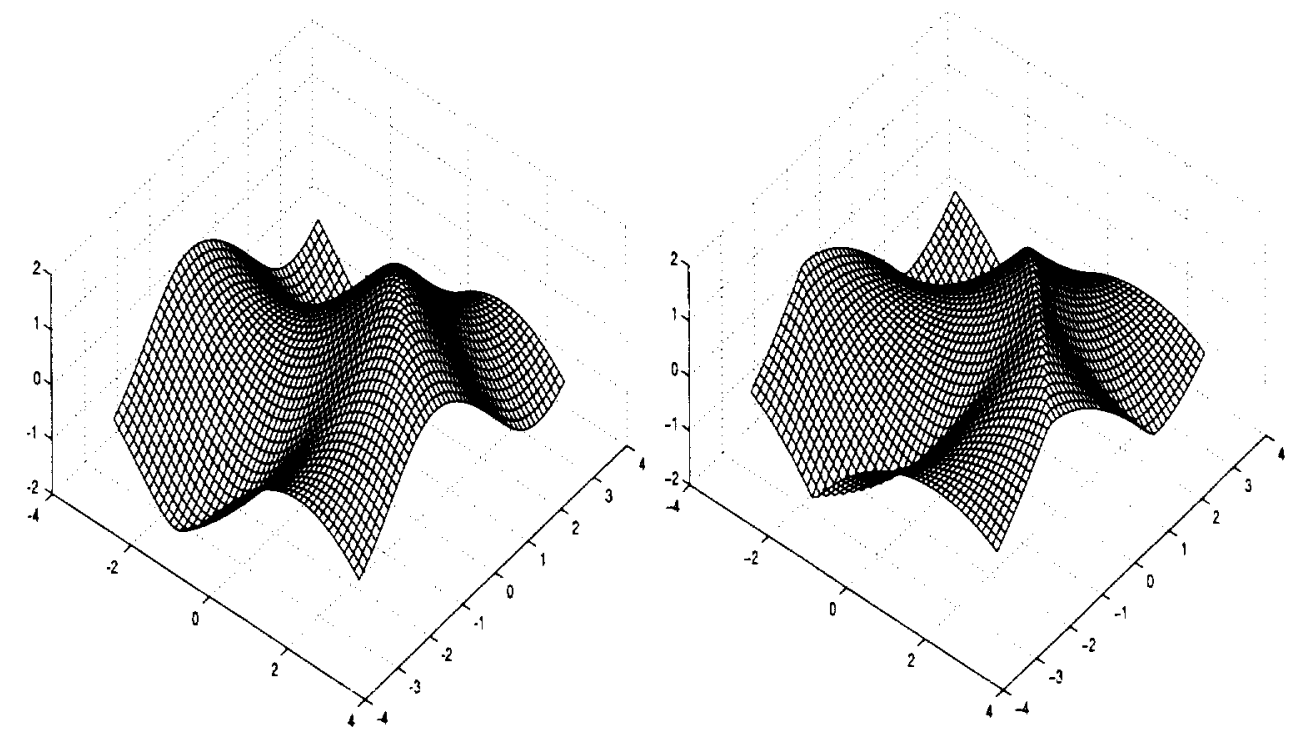

Figure 4.7: Fully two-dimensional Hamiltonian, (4.6). Left: the solution before the singularity formation, $T=0.8$. Right: the solution after the singularity formation, $T=1.5 . \quad N=50 \times 50$. The solution is computed with (3.9) integrated in time as in Algorithm 2.1, with the fifth-order reconstruction of Section 3.3.

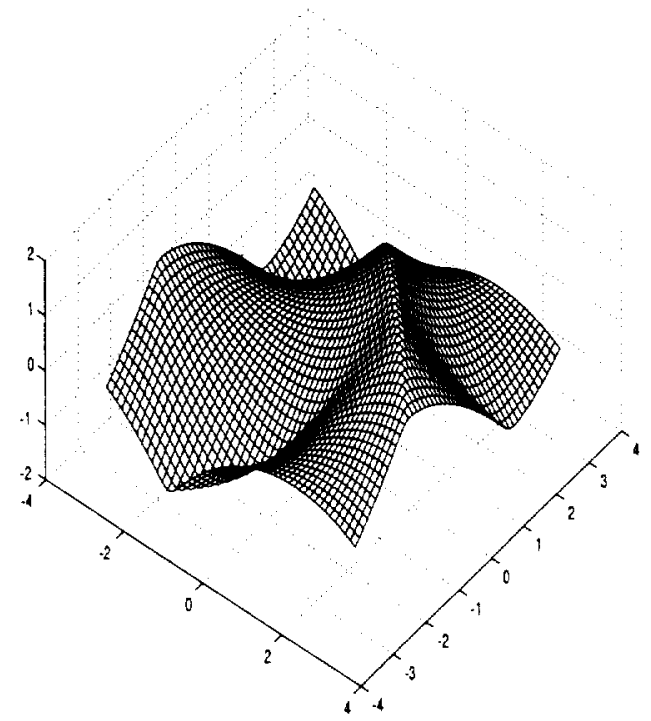

Figure 4.8: The fully two-dimensional Hamiltonian computed with the method in [6] after the singularity formation, $T=1.5 . N=50 \times 50$. 

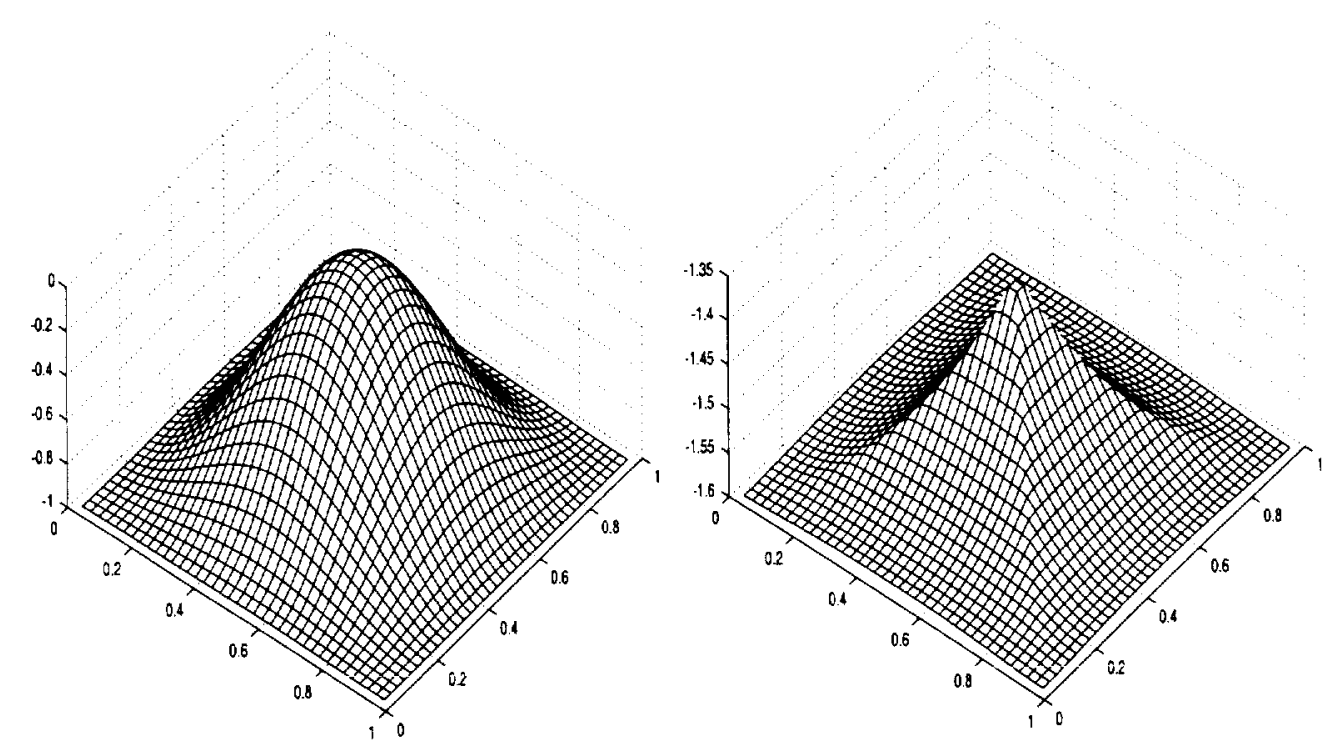

Figure 4.9: Two-dimensional eikonal equation, (4.7). $N=40 \times 40$. Left: the initial data. Right: our semi-discrete fifth-order semi-discrete approximation at $T=0.6$.

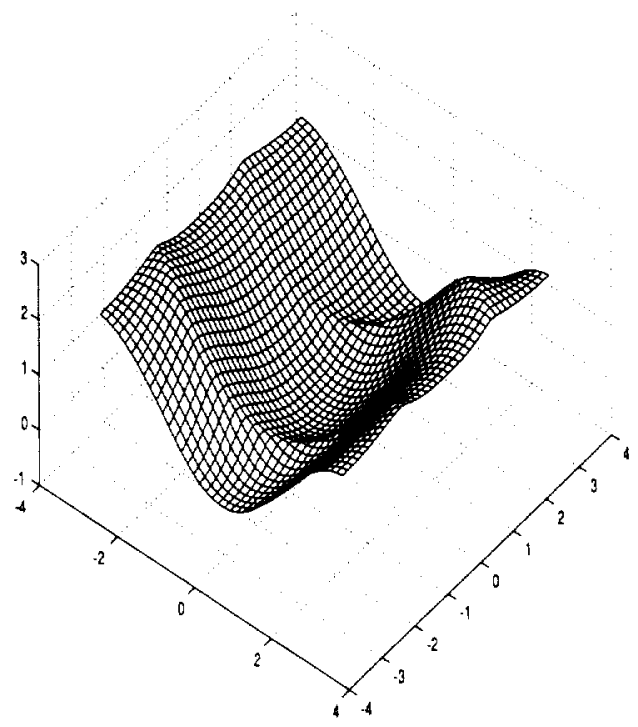

Figure 4.10: Two-dimensional optimal control problem, (4.8). An approximation with our semi-discrete fifth-order method (3.9) is shown at $T=1 . N=40 \times 40$. 


\subsection{A Stability Study}

In this section we present a stability study, checking the stability properties of the two-dimensional semi-discrete fifth-order method. We compute the relative $L^{1}$ errors for various examples while varying the CFL number. In Figure 4.11 we compare the results obtained with our fifth-order scheme with the fully-discrete method [6], and with the upwind method of [15] using a local Lax-Friedrichs flux. As expected, the stability properties of the method (3.9) are similar to the stability properties of the upwind WENO method of [15], though our new method (3.9) enjoys smaller $L^{1}$ errors and hence is more accurate.

\subsection{Three-Dimensional Examples}

Finally, we solve a couple of three-dimensional problems with the scheme (3.11) integrated in time as in Algorithm 2.1, with the fifth-order reconstruction (3.13). We start with a convex problem

$$
\phi_{t}+\frac{1}{2}\left(\phi_{x}+\phi_{y}+\phi_{z}+1\right)^{2}=0
$$

subject to the initial data $\phi(x, y, z, 0)=-\cos (\pi(x+y+z) / 3)$. The convergence results for the scheme (3.11) before and after the singularity formation are given in Table 4.6. We also use (3.11) to approximate the solution of the non-covex problem

$$
\phi_{t}-\cos \left(\phi_{x}+\phi_{y}+\phi_{z}+1\right)=0
$$

with the same initial data. The convergence rates are shown in Table 4.7.

\begin{tabular}{|c||c|c||c|c||}
\hline \multicolumn{5}{|c|}{ Before singularity $T=0.5 / \pi^{2}$} \\
\hline$N$ & relative $L^{1}$-error & $L^{1}$-order & relative $L^{\infty}$-error & $L^{\infty}$-order \\
\hline \hline 25 & $1.04 \times 10^{-4}$ & - & $3.10 \times 10^{-8}$ & - \\
\hline 50 & $6.52 \times 10^{-6}$ & 3.99 & $2.66 \times 10^{-10}$ & 6.87 \\
\hline 100 & $3.74 \times 10^{-7}$ & 4.12 & $2.02 \times 10^{-12}$ & 7.04 \\
\hline \multicolumn{5}{|c|}{ After singularity $T=1.5 / \pi^{2}$} \\
\hline$N$ & relative $L^{1}$-error & $L^{1}$-order & relative $L^{\infty}$-error & $L^{\infty}$-order \\
\hline \hline 25 & $1.40 \times 10^{-3}$ & - & $9.76 \times 10^{-6}$ & - \\
\hline 50 & $1.80 \times 10^{-4}$ & 2.95 & $4.15 \times 10^{-6}$ & 1.23 \\
\hline 100 & $1.26 \times 10^{-4}$ & 0.51 & $6.94 \times 10^{-7}$ & 2.58 \\
\hline
\end{tabular}

Table 4.6: Relative $L^{1}$ - and $L^{\infty}$-errors for the three-dimensional convex HJ problem (4.9) before and after the singularity formation, computed with (3.11) integrated in time as in Algorithm 2.1, with the fifth-order reconstruction (3.13). 

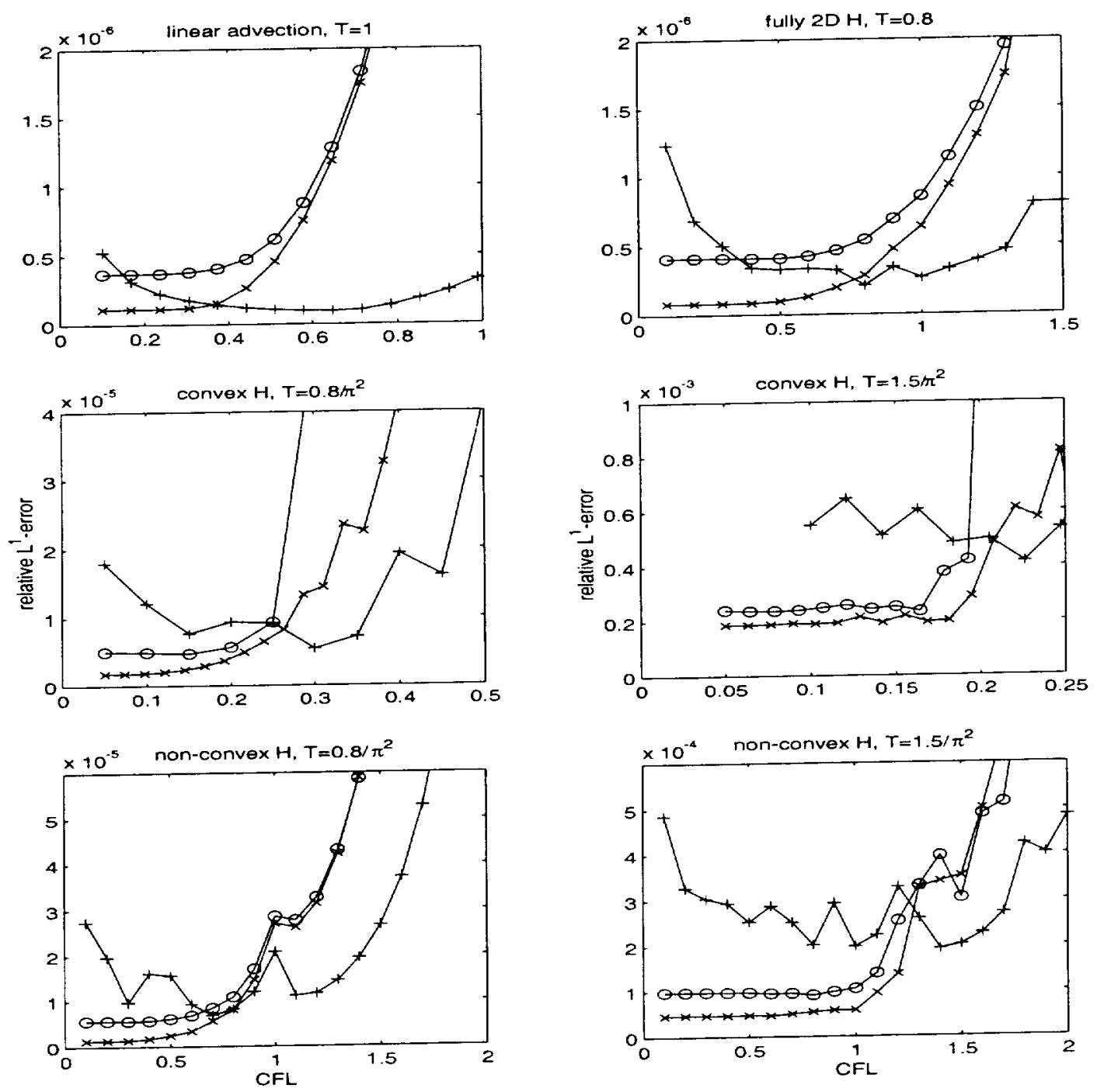

Figure 4.11: Stability of the two-dimensional semi-discrete methods. $N=100 \times 100$. "x": our semi-discrete fifth-order method (3.9). "+": our fully-discrete fifth-order method [6]. "o": the fifth-order upwind method of [15] with a local Lax-Friedrichs flux. Upper left: linear advection with initial condition $\phi(x, y, 0)=-\cos (\pi(x+y) / 2)$. Upper right: fully 2D Hamiltonian (4.6). Middle row: convex Hamiltonian (4.4), before the singularity (left) and after the singularity (right). Bottom row: non-convex Hamiltonian (4.5), before the singularity (left) and after the singularity (right). 


\begin{tabular}{|c||c|c||c|c||}
\hline \multicolumn{5}{|c||}{ Before singularity $T=0.5 / \pi^{2}$} \\
\hline$N$ & relative $L^{1}$-error & $L^{1}$-order & relative $L^{\infty}$-error & $L^{\infty}$-order \\
\hline \hline 25 & $9.10 \times 10^{-5}$ & - & $2.58 \times 10^{-8}$ & - \\
\hline 50 & $3.85 \times 10^{-6}$ & 4.56 & $2.27 \times 10^{-10}$ & 6.83 \\
\hline 100 & $1.77 \times 10^{-7}$ & 4.45 & $1.53 \times 10^{-12}$ & 7.21 \\
\hline \multicolumn{5}{|c||}{ After singularity $T=1.5 / \pi^{2}$} \\
\hline$N$ & relative $L^{1}$-error & $L^{1}$-order & relative $L^{\infty}$-error & $L^{\infty}$-order \\
\hline \hline 25 & $9.99 \times 10^{-4}$ & - & $6.60 \times 10^{-7}$ & - \\
\hline 50 & $1.09 \times 10^{-4}$ & 3.20 & $5.25 \times 10^{-7}$ & 0.33 \\
\hline 100 & $1.07 \times 10^{-5}$ & 3.34 & $6.13 \times 10^{-8}$ & 3.01 \\
\hline
\end{tabular}

Table 4.7: Relative $L^{1}$ - and $L^{\infty}$-errors for the three-dimensional non-convex $\mathrm{HJ}$ problem (4.10) before and after the singularity formation, computed with (3.11) integrated in time as in Algorithm 2.1, with the fifth-order reconstruction (3.13).

\section{Appendix A: A Proof of Theorem 2.1}

Proof. Let $H(u) \in C^{2}$ be convex $\left(H^{\prime \prime}(u) \geq 0\right.$ or $\left.H^{\prime \prime}(u) \leq 0\right)$. We need to show that the flux

$$
H^{K N P}\left(u^{+}, u^{-}\right)=\frac{1}{a^{+}+a^{-}}\left[a^{-} H\left(u^{+}\right)+a^{+} H\left(u^{-}\right)\right]-\frac{a^{+} a^{-}}{a^{+}+a^{-}}\left(u^{+}-u^{-}\right),
$$

is a non-increasing function of $u^{+}$and a non-decreasing function of $u^{-}$. Here $a^{+}$and $a^{-}$are defined as

$$
a^{+}=\max _{u \in I\left(u^{-}, u^{+}\right)}\left\{H^{\prime}(u), 0\right\}, a^{-}=\min _{u \in I\left(u^{-}, u^{+}\right)}\left|\left\{H^{\prime}(u), 0\right\}\right|,
$$

where $I(a, b)$ is the closed interval with endpoints $a$ and $b$.

The proof for $u^{+}$is discussed in detail. The proof for $u^{-}$is similar.

Let $u_{1}^{+}>u_{2}^{+}$. Define the difference

$$
\begin{aligned}
D= & H^{K N P}\left(u_{1}^{+}, u^{-}\right)-H^{K N P}\left(u_{2}^{+}, u^{-}\right) \\
= & \frac{1}{a_{1}^{+}+a_{1}^{-}}\left[a_{1}^{-} H\left(u_{1}^{+}\right)+a_{1}^{+} H\left(u^{-}\right)\right]-\frac{a_{1}^{+} a_{1}^{-}}{a_{1}^{+}+a_{1}^{-}}\left(u_{1}^{+}-u^{-}\right) \\
& -\frac{1}{a_{2}^{+}+a_{2}^{-}}\left[a_{2}^{-} H\left(u_{2}^{+}\right)+a_{2}^{+} H\left(u^{-}\right)\right]+\frac{a_{2}^{+} a_{2}^{-}}{a_{2}^{+}+a_{2}^{-}}\left(u_{2}^{+}-u^{-}\right) .
\end{aligned}
$$

We will prove that $D \leq 0$. We rewrite the $D$ as the difference

$$
D=G\left(u_{1}^{+}\right)-G\left(u_{2}^{+}\right) \text {, }
$$

where for fixed $u^{-}$

$$
G(u)=A(u)\left[H(u)-H\left(u^{-}\right)-a^{+}(u)\left(u-u^{-}\right)\right],
$$


with

$$
A(u)=\frac{a^{-}(u)}{a^{+}(u)+a^{-}(u)}, a^{+}(u)=\max _{u \in I\left(u^{-}, u\right)}\left\{H^{\prime}(u), 0\right\}, a^{-}(u)=\min _{u \in I\left(u^{-}, u\right)}\left|\left\{H^{\prime}(u), 0\right\}\right| .
$$

Since $u_{1}^{+}>u_{2}^{+}$, the requirement $D \leq 0$ is equivalent to $G^{\prime}(u) \leq 0$. Because $H$ is convex (so the extrema of $H^{\prime}$ on an interval occur at the endpoints of that interval), $a^{ \pm} \in C^{1}$ so

$$
\begin{aligned}
G^{\prime}(u)= & A^{\prime}(u)\left[H(u)-H\left(u^{-}\right)-a^{+}(u)\left(u-u^{-}\right)\right] \\
& +A(u)\left[H^{\prime}(u)-a^{+\prime}(u)\left(u-u^{-}\right)-a^{+}(u)\right]
\end{aligned}
$$

Because $H$ is continuous, there exists a $\xi(u) \in I\left(u^{-}, u\right)$ such that $H^{\prime}(\xi(u))\left(u-u^{-}\right)=H(u)-H\left(u^{-}\right)$so

$$
\begin{aligned}
G^{\prime}(u)= & A^{\prime}(u)\left(H^{\prime}(\xi(u))-a^{+}(u)\right)\left(u-u^{-}\right)-A(u) a^{+\prime}(u)\left(u-u^{-}\right) \\
& +A(u)\left(H^{\prime}(u)-a^{+}(u)\right) \\
= & B(u)\left(u-u^{-}\right)+A(u)\left(H^{\prime}(u)-a^{+}(u)\right)
\end{aligned}
$$

where $B(u)=A^{\prime}(u)\left(H^{\prime}(\xi(u))-a^{+}(u)\right)-A(u) a^{+\prime}(u)$.

Now

$$
A^{\prime}(u)=\frac{a^{+}(u) a^{-\prime}(u)-a^{-}(u) a^{+\prime}(u)}{\left(a^{+}(u)+a^{-}(u)\right)^{2}}
$$

hence

$$
\begin{aligned}
B(u)= & \frac{1}{\left(a^{+}(u)+a^{-}(u)\right)^{2}}\left[a^{+}(u) a^{-\prime}(u)\left(H^{\prime}(\xi(u))-a^{+}(u)\right)\right. \\
& \left.-a^{-}(u) a^{+\prime}(u)\left(H^{\prime}(\xi(u))+a^{-}(u)\right)\right] .
\end{aligned}
$$

We are now in a position to prove that $D \leq 0$. There are three cases to consider.

Case 1. $u_{1}^{+}>u_{2}^{+} \geq u^{-}$. In this case $u-u^{-} \geq 0$, and if $u_{1}>u_{2}$ then $\left[u^{-}, u_{1}\right] \supset\left[u^{-}, u_{2}\right]$ so $a^{ \pm}\left(u_{1}\right) \geq a^{ \pm}\left(u_{2}\right)$ and $a^{ \pm \prime}(u) \geq 0$. Then

$$
\begin{aligned}
B(u)= & \frac{1}{\left(a^{+}(u)+a^{-}(u)\right)^{2}}[\underbrace{a^{+}(u) a^{-1}(u)}_{\geq 0} \underbrace{\left(H^{\prime}(\xi(u))-a^{+}(u)\right)}_{\leq 0} \\
& -\underbrace{a^{-}(u) a^{+1}(u)}_{\geq 0} \underbrace{\left(H^{\prime}(\xi(u))+a^{-}(u)\right)}_{\geq 0}] \leq 0,
\end{aligned}
$$

so

$$
G^{\prime}(u)=\underbrace{B(u)}_{\leq 0} \underbrace{\left(u-u^{-}\right)}_{\geq 0}+\underbrace{A(u)}_{\geq 0} \underbrace{\left(H^{\prime}(u)-a^{+}(u)\right)}_{\leq 0} \leq 0 .
$$

Case 2. $u^{-} \geq u_{1}^{+}>u_{2}^{+}$. In this case $u-u^{-} \leq 0$, and if $u_{1}>u_{2}$ then $\left[u^{-}, u_{1}\right] \subset\left[u^{-}, u_{2}\right]$ so $a^{ \pm}\left(u_{1}\right) \leq a^{ \pm}\left(u_{2}\right)$ and $a^{ \pm \prime}(u) \leq 0$. We therefore have $B(u) \geq 0$ and

$$
G^{\prime}(u)=\underbrace{B(u)}_{\geq 0} \underbrace{\left(u-u^{-}\right)}_{\leq 0}+\underbrace{A(u)}_{\geq 0} \underbrace{\left(H^{\prime}(u)-a^{+}(u)\right)}_{\leq 0} \leq 0 .
$$


Case 3. $u_{1}^{+}>u^{-} \geq u_{2}^{+}$. In this case the proof is much more direct: by the continuity of $H$ there exists a $\xi_{1} \in\left[u^{-}, u_{1}^{+}\right]$and a $\xi_{2} \in\left[u_{2}^{+}, u^{-}\right]$such that

$$
\begin{aligned}
D & =\frac{a_{1}^{-}}{a_{1}^{+}+a_{1}^{-}}\left(H^{\prime}\left(\xi_{1}\right)-a_{1}^{+}\right)\left(u_{1}^{+}-u^{-}\right)-\frac{a_{2}^{-}}{a_{2}^{+}+a_{2}^{-}}\left(H^{\prime}\left(\xi_{2}\right)-a_{2}^{+}\right)\left(u_{2}^{+}-u^{-}\right) \\
& \leq 0 .
\end{aligned}
$$

The proof that $H^{K N P}\left(u^{+}, u^{-}\right)$is non-decreasing in $u^{-}$is the same with

$$
G(u)=\frac{a^{+}(u)}{a^{+}(u)+a^{-}(u)}\left[H(u)-H\left(u^{+}\right)+a^{-}(u)\left(u-u^{+}\right)\right]
$$

for fixed $u^{+}$.

\section{References}

[1] Abgrall R., Numerical discretization of the first-order Hamilton-Jacobi equation on triangular meshes, Comm. Pure Appl. Math., 49 (1996), pp.1339-1373.

[2] Barles G., Solution de viscosité des équations de Hamilton-Jacobi, Springer-Verlag, Berlin, 1994.

[3] Bianco F., Puppo G., Russo G., High order central schemes for hyperbolic systems of conservation laws, SIAM J. Sci. Comp., 21 (1999), pp.294-322.

[4] Bryson S., Levy D., Central schemes for multi-dimensional Hamilton-Jacobi equations, NAS Technical Report NAS-01-014, 2001, SIAM J. Sci. Comp., to appear.

[5] Bryson S., Levy D., High-order central WENO schemes for $1 D$ Hamilton-Jacobi equations, Proc. Enumath 2001, Ischia, Italy, to appear.

[6] Bryson S., Levy D., High-order central WENO schemes for multi-dimensional Hamilton-Jacobi equations, NAS Technical Report NAS-02-004, submitted to SIAM J. Numer. Anal.

[7] Crandall M.G., Evans L.C., Lions P.-L., Some properties of viscosity solutions of Hamilton-Jacobi equations, Trans. Amer. Math. Soc., 282 (1984), pp.487-502.

[8] Crandall M.G., Ishii H., Lions P.-L., User's guide to viscosity solutions of second order partial differential equations, Bull. Amer. Math. Soc., 27 (1992), pp.1-67.

[9] Crandall M.G., Lions P.-L., Viscosity solutions of Hamilton-Jacobi equations, Trans. Amer. Math. Soc., 277 (1983), pp.1-42.

[10] Crandall M.G., Lions P.-L., Two approximations of solutions of Hamilton-Jacobi equations, Math. Comp., 43 (1984), pp.1-19. 
[11] Friedrichs K.O., Lax P.D., Systems of conservation equations with a convex extension, Proc. Nat. Acad. Sci., 68 (1971), pp.1686-1688.

[12] Gottlieb S., Shu C.-W., Tadmor E., Strong stability-preserving high order time discretization methods, SIAM Review, 43 (2001), pp.89-112.

[13] Harten A., Engquist B., Osher S., Chakravarthy S., Uniformly high order accurate essentially non-oscillatory schemes III, J. Comp. Phys., 71 (1987), pp.231-303.

[14] Kruzkov S.N., The Cauchy problem in the large for nonlinear equations and for certain quasilinear systems of the first order with several variables, Soviet Math. Dokl., 5 (1964), pp.493-496.

[15] Jiang G.-S., Peng D., Weighted ENO schemes for Hamilton-Jacobi equations, SIAM J. Sci. Comp., 21 (2000), pp.2126-2143.

[16] Jiang G.-S., Shu C.-W., Efficient implementation of weighted ENO schemes, J. Comp. Phys., 126 (1996), pp.202-228.

[17] Jin S., Xin Z., Numerical passage from systems of conservation laws to HamiltonJacobi equations and relaxation schemes, SIAM J. Numer. Anal., 35 (1998), pp.23852404.

[18] Kurganov A., Levy D., A Third-Order Semi-Discrete Scheme for Conservation Laws and Convection-Diffusion Equations, SIAM J. Sci. Comp., 22, (2000), pp.14611488.

[19] Kurganov A., Noelle S., Petrova G., Semi-discrete central-upwind schemes for hyperbolic conservation laws and Hamilton-Jacobi equations, SIAM J. Sci. Comp., 23 (2001), pp.707-740.

[20] Kurganov A., Tadmor E., New high-resolution semi-discrete central schemes for Hamilton-Jacobi equations, J. Comp. Phys., 160 (2000), pp.720-742.

[21] Kurganov A., Tadmor E., New high-resolution central schemes for nonlinear conservation laws and convection-diffusion equations, J. Comp. Phys., 160 (2000), pp.241282.

[22] Levy D., Puppo G., Russo G., A fourth order central WENO scheme for multidimensional hyperbolic systems of conservation laws, SIAM J. Sci. Comp., to appear.

[23] Levy D., Puppo G., Russo G., Central WENO schemes for hyperbolic systems of conservation laws, Math. Model. and Numer. Anal., 33, no. 3 (1999), pp.547-571.

[24] Levy D., Puppo G., Russo G., Compact central WENO schemes for multidimensional conservation laws, SIAM J. Sci. Comp., 22 (2000), pp.656-672.

[25] Lions P.L., Generalized solutions of Hamilton-Jacobi equations, Pitman, London, 1982. 
[26] Lions P.L., Souganidis P.E., Convergence of MUSCL and filtered schemes for scalar conservation laws and Hamilton-Jacobi equations, Numer. Math., 69 (1995), pp.441470 .

[27] Lin C.-T., Tadmor E., $L^{1}$-stability and error estimates for approximate HamiltonJacobi solutions, Numer. Math., 87 (2001), pp.701-735.

[28] Lin C.-T., Tadmor E., High-resolution non-oscillatory central schemes for approximate Hamilton-Jacobi equations, SIAM J. Sci. Comp., 21, no. 6 (2000), pp.21632186.

[29] Liu X.-D., Osher S., Chan T., Weighted essentially non-oscillatory schemes, J. Comp. Phys., 115 (1994), pp.200-212.

[30] Nessyahu H., Tadmor E., Non-oscillatory central differencing for hyperbolic conservation laws, J. Comp. Phys., 87, no. 2 (1990), pp.408-463.

[31] Osher S., Sethian J., Fronts propagating with curvature dependent speed: algorithms based on Hamilton-Jacobi formulations, J. Comp. Phys., 79 (1988), pp.12-49.

[32] Osher S., Shu C.-W., High-order essentially nonoscillatory schemes for HamiltonJacobi equations, SIAM J. Numer. Anal., 28 (1991), pp.907-922.

[33] Souganidis P.E., Approximation schemes for viscosity solutions of Hamilton-Jacobi equations, J. Diff. Equations., 59 (1985), pp.1-43.

[34] Zhang Y.-T., Shu C.-W., High-order WENO schemes for Hamilton-Jacobi equations on triangular meshes, NASA/CR-2001-211256, ICASE Report No. 2001-39, (2001), submitted to SIAM J. Sci. Comp. 